\title{
A free boundary problem for an attraction-repulsion chemotaxis system
}

Weiyi Zhang', Zuhan Liu' and Ling Zhou'*

\section{${ }^{*}$ Correspondence:}

zhoul@yzu.edu.cn

'School of Mathematical Science,

Yangzhou University, Yangzhou,

China

\begin{abstract}
In this paper we study an attraction-repulsion chemotaxis system with a free boundary in one space dimension. First, under some conditions, we investigate existence, uniqueness and uniform estimates of the global solution. Next, we prove a spreading-vanishing dichotomy for this model. In the vanishing case, the species fail to establish and die out in the long run. In the spreading case, we provide some sufficient conditions to prove that the species successfully spread to infinity as $t \rightarrow \infty$ and stabilize at a constant equilibrium state. The criteria for spreading and vanishing are also obtained.
\end{abstract}

MSC: 35K51; 35R35; 92B05; 35B40

Keywords: Attraction-repulsion chemotaxis system; Free boundary; Spreading and vanishing

\section{Introduction}

This paper is devoted to studying the dynamics of solutions to the following attractionrepulsion chemotaxis system with a free boundary:

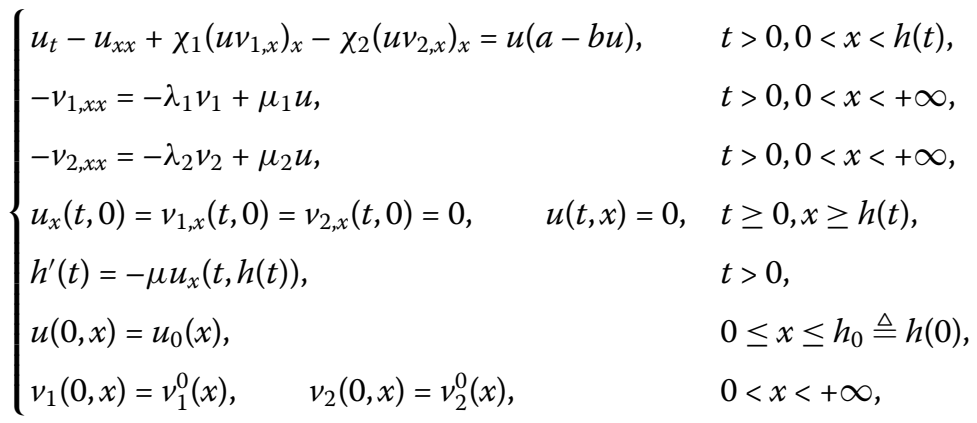

where $u(t, x)$ represents the population density of mobile species, $v_{1}(t, x)$ represents the population density of a chemoattractant, $v_{2}(t, x)$ represents the population density of a chemorepulsion. The right-hand side of the first equation in (1.1) is a logistic reaction term $u(a-b u)$. The coefficient $a$ represents the intrinsic growth rate of the cells, $b$ measures its intraspecific competition, $\chi_{1} \geq 0$ and $\chi_{2} \geq 0$ represent the chemotaxis, and the positive constants $\lambda_{1}, \lambda_{2}, \mu_{1}$ and $\mu_{2}$ are related to growth rate of the chemical substances. The

(c) The Author(s) 2018. This article is distributed under the terms of the Creative Commons Attribution 4.0 International License (http://creativecommons.org/licenses/by/4.0/), which permits unrestricted use, distribution, and reproduction in any medium, provided you give appropriate credit to the original author(s) and the source, provide a link to the Creative Commons license, and indicate if changes were made. 
initial functions $u_{0}(x), v_{i}^{0}(x)$ satisfy

$$
\left\{\begin{array}{l}
u_{0} \in C^{2}\left(\left[0, h_{0}\right]\right), \quad u_{0}\left(h_{0}\right)=u_{0}^{\prime}(0)=0, \quad u_{0}>0 \quad \text { in }\left[0, h_{0}\right), \\
v_{i}^{0} \in C_{b}([0,+\infty)), \quad v_{i}^{0}(0)=0, \\
v_{i}^{0}(x) \not \equiv 0, \quad v_{i}^{0}(x) \geq 0 \quad \text { in }[0,+\infty), i=1,2,
\end{array}\right.
$$

here $C_{b}([0,+\infty))$ is the space of continuous and bounded functions in $[0,+\infty)$.

The free boundary $x=h(t)$ represents the spreading front, the homogeneous Neumann boundary condition at $x=0$ indicates that the left boundary is fixed and no species cross the left boundary, while the species only spreads to the right-hand side. The equation governing the free boundary, $h^{\prime}(t)=-\mu u_{x}(t, h(t))$, is a special case of the well-known Stefan condition arising from the investigation of the melting of ice in contact with water [26]. At present, it was used to describe the invasion of species [22, 23].

When $h(t) \equiv$ const. and $\chi_{2}=\mu_{2}=\lambda_{2}=0$, system (1.1) is a classical model of chemotaxis in one dimension $n=1$, which was first introduced by Keller and Segel in [16, 17]. In the case $a=b=0$, this system is proved to have finite time blowing up solutions for large enough initial conditions in dimensions $n \geq 2$ (see [24,25]), but for $n=1$ there is no blow up. Since the works by Keller and Segel, a rich variety of mathematical models for studying chemotaxis have appeared (see [1, 24, 25, 27-29, 45] and the references therein). In [28], Salako and Shen considered an attraction-repulsion chemotaxis systems with proliferation and death of cells and assumed that chemicals diffuse very quickly. They investigated the global existence of classical solutions, stability of constant equilibria and spreading speed of attraction-repulsion chemotaxis systems with a logistic source on $\mathbb{R}^{N}$. Salako and Shen [29] have further studied traveling wave solutions of the attraction-repulsion chemotaxis system. Moreover, attraction-repulsion chemotaxis systems on bounded domains have been studied in many papers (see $[14,20,21,43,44,50]$ and the references therein).

In many realistic modeling situations, species have a tendency to emigrate from the boundary, to obtain their new habitat and improve the living environment. Hence, it is more reasonable to consider the domain with a free boundary. Motivated by the work of Du and Lin [7], there have been many theoretical developments on the free boundary problem in various environments (see $[2,4-6,8,10-13,18,19,30-32,34,35,40,41,46,47$, $49,51])$. For example, the authors of $[5,35]$ dealt with the equation with a free boundary in time-periodic environment. The authors of $[4,6]$ considered the higher dimensions' and heterogeneous environment case, while the authors of [31,32, 34, 41, 46, 51] have studied two species predator-prey models with a free boundary. Wang and Wang [31] considered the diffusive Beddington-DeAngelis predator-prey model with nonlinear prey-taxis and free boundary. To the best of our knowledge, this is the first work concerning the free boundary problem of chemotaxis systems. Other works for various species competition systems with free boundaries can be found in $[8,10,12,13,40,47,49]$. The authors of [11, $18,19,30]$ considered the transmission of viruses and diseases by using a free boundary. For the study of free boundary problems for other biological models, please refer to [3-7, $9,15,33,35,36,48,52]$ and the references therein.

The main intention of this paper is to analyze the dynamical behavior of model (1.1) when both chemo-attraction and chemo-repulsion are present. Compared with [28], due to the influence of the free boundary, we derive different dynamic behaviors of species. 
Precisely, we prove a spreading-vanishing dichotomy for this model, that is, the species either fails to establish and vanishes eventually (called vanishing) or the species successfully spreads to infinity as $t \rightarrow \infty$ and stabilizes at a constant equilibrium state under some sufficient conditions (called spreading). In other words, the solution $\left(u, v_{1}, v_{2}, h\right)$ of (1.1) exhibits the following behavior:

(i) Vanishing: if $\lim _{t \rightarrow+\infty} h(t)<\infty$, then we have $\lim _{t \rightarrow+\infty}\|u(t, x)\|_{C([0, h(t)])}=0$, $\lim _{t \rightarrow+\infty} v_{i}(t, x)=0, i=1,2$, uniformly in $[0,+\infty)$.

(ii) Spreading: if $\lim _{t \rightarrow+\infty} h(t)=\infty$,

$$
b>\chi_{1} \mu_{1}-\chi_{2} \mu_{2}+K
$$

where

$$
\begin{aligned}
K:= & \min \left\{\frac{1}{\lambda_{2}}\left(\left|\chi_{1} \mu_{1} \lambda_{1}-\chi_{2} \mu_{2} \lambda_{2}\right|+\chi_{1} \mu_{1}\left|\lambda_{1}-\lambda_{2}\right|\right),\right. \\
& \left.\frac{1}{\lambda_{1}}\left(\left|\chi_{1} \mu_{1} \lambda_{1}-\chi_{2} \mu_{2} \lambda_{2}\right|+\chi_{2} \mu_{2}\left|\lambda_{1}-\lambda_{2}\right|\right)\right\},
\end{aligned}
$$

then we have

$$
\lim _{t \rightarrow \infty} u(t, x)=\frac{a}{b}, \quad \lim _{t \rightarrow \infty} v_{i}(t, x)=\frac{\mu_{i}}{\lambda_{i}} \frac{a}{b}, \quad i=1,2,
$$

uniformly in any bounded subset of $[0,+\infty)$.

For the spreading case, condition (1.3) is similar to that in [28]; however, we use different approaches compared to [28] to obtain this convergence result, due to different boundary conditions. In mathematics, it is difficult to find a pair of upper and lower solutions at once to squeeze the solution of problem (1.1). To overcome the difficulty, we first prove Lemmas 3.4-3.5 which will be used to establish the main Theorem 3.6. Second, we construct a super/sub-solution to compare with the solution of (1.1). Using an iterative method, we construct more suitable upper and lower solution sequences $\left\{\bar{u}_{i}\right\}$ and $\left\{\underline{u}_{i}\right\}$. Finally, by the monotone convergence theorem, we derive the exact long time behavior of the solution. We also prove that the limits of sequences $\left\{\underline{u}_{i}\right\}$ and $\left\{\bar{u}_{i}\right\}$ both converge to $a / b$, which involves applying some new ideas and techniques (see Theorem 3.6).

The organization of this paper is as follows. In Sect. 2, we give the proof of the global existence, uniqueness and estimates of $\left(u, v_{1}, v_{2}, h\right)$. Section 3 is devoted to the long time behavior of $\left(u, v_{1}, v_{2}\right)$. In Sect. 4 , we give some sufficient conditions of spreading and vanishing. The paper ends with a brief conclusion.

\section{Global existence, uniqueness and estimates of the solution}

In this section, we give the existence and uniqueness results. The proof of the local existence and uniqueness can be done by modifying the arguments of [36]. Define the kernel function

$$
\phi_{i}(t, x) \triangleq \frac{\sqrt{\lambda_{i}}}{2} e^{-\sqrt{\lambda_{i}}|x|}, \quad i=1,2
$$


If $\left(u, v_{1}, v_{2}, h\right)$ is a solution of $(1.1)$, then

$$
\begin{aligned}
v_{i}(t, x) & =\frac{\mu_{i}}{\lambda_{i}}\left(\phi_{i} * u\right)(x) \\
& :=\frac{\mu_{i}}{\lambda_{i}}\left[\int_{-\infty}^{0} \phi_{i}(x-y) u(t,-y) d y+\int_{0}^{+\infty} \phi_{i}(x-y) u(t, y) d y\right], \quad x \in \mathbb{R}^{+} .
\end{aligned}
$$

Thus equation (1.1) is equivalent to the following problem:

$$
\begin{cases}u_{t}-u_{x x}+\frac{\chi_{1} \mu_{1}}{\lambda_{1}}\left(\phi_{1} * u\right)_{x} u_{x}-\frac{\chi_{2} \mu_{2}}{\lambda_{2}}\left(\phi_{2} * u\right)_{x} u_{x} & \\ \quad=u\left[a-\left(b+\chi_{2} \mu_{2}-\chi_{1} \mu_{1}\right) u\right. & \\ \left.\quad-\chi_{1} \mu_{1}\left(\phi_{1} * u\right)+\chi_{2} \mu_{2}\left(\phi_{2} * u\right)\right], & t>0,0<x<h(t), \\ u_{x}(t, 0)=0, \quad u(t, x)=0, & t \geq 0, x \geq h(t), \\ h^{\prime}(t)=-\mu u_{x}(t, h(t)), & t>0, \\ u(0, x)=u_{0}(x), & 0 \leq x \leq h_{0} \triangleq h(0) .\end{cases}
$$

Lemma 2.1 For any given $\theta \in(0,1), p \geq 3 /(1-\theta)$ and

$$
b>\chi_{1} \mu_{1}-\chi_{2} \mu_{2}+H \text {, }
$$

where

$$
H:=\min \left\{H_{1}, H_{2}\right\}
$$

with

$$
\left\{\begin{array}{l}
H_{1}=\frac{1}{\lambda_{1}}\left[\left(\chi_{2} \mu_{2} \lambda_{2}-\chi_{1} \mu_{1} \lambda_{1}\right)_{+}+\chi_{2} \mu_{2}\left(\lambda_{1}-\lambda_{2}\right)_{+}\right], \\
H_{2}=\frac{1}{\lambda_{2}}\left[\left(\chi_{2} \mu_{2} \lambda_{2}-\chi_{1} \mu_{1} \lambda_{1}\right)_{+}+\chi_{1} \mu_{1}\left(\lambda_{1}-\lambda_{2}\right)_{+}\right],
\end{array}\right.
$$

the following is true:

(i) There exists a $T>0$ such that problem (1.1) admits a unique solution

$$
\begin{aligned}
\left(u, v_{1}, v_{2}, h\right) \in & W_{p}^{1,2}\left(D_{T}\right) \cap C^{(1+\theta) / 2,1+\theta}\left(\bar{D}_{T}\right) \times\left[C^{(1+\theta) / 2,1+\theta}\left(D_{v}^{T}\right)\right]^{2} \\
& \times C^{1+\theta / 2}([0, T])
\end{aligned}
$$

where $D_{T}:=\left\{(t, x) \in \mathbb{R}^{2}: t \in(0, T), x \in[0, h(t)]\right\}$, $D_{v}^{T}:=\left\{(t, x) \in \mathbb{R}^{2}: t \in(0, T), x \in[0, \infty)\right\}$, and $C$ is independent of $T$.

(ii) Let $0<\tau<\infty$ and $\left(u, v_{1}, v_{2}, h\right) \in W_{p}^{1,2}\left(D_{\tau}\right) \cap C^{(1+\theta) / 2,1+\theta}\left(\bar{D}_{\tau}\right) \times\left[C^{(1+\theta) / 2,1+\theta}\left(D_{v}^{\tau}\right)\right]^{2} \times$ $C^{1+\theta / 2}([0, \tau])$ be the unique solution of (1.1). Then we have

$$
\begin{aligned}
0 & <u(t, x) \\
& \leq \max \left\{\frac{a}{b+\chi_{2} \mu_{2}-\chi_{1} \mu_{1}-H},\left\|u_{0}\right\|_{L^{\infty}}\right\} \\
& \triangleq R_{1} \quad \text { for } t \in(0, \tau), x \in[0, h(t)),
\end{aligned}
$$




$$
\begin{aligned}
0< & v_{i}(t, x) \\
\leq & \max \left\{\frac{\mu_{i}}{\lambda_{i}} \frac{a}{b+\chi_{2} \mu_{2}-\chi_{1} \mu_{1}-H}, \frac{\mu_{i}}{\lambda_{i}}\left\|u_{0}\right\|_{L^{\infty}}\right\} \\
& \text { for } t \in(0, \tau), \quad x \in[0,+\infty), \\
0< & h^{\prime}(t) \leq C \text { for } t \in(0, \tau),
\end{aligned}
$$

where the positive constant $C$ is independent of $\tau$.

Proof As in [36], we first straighten the free boundary and define

$$
y=\frac{x}{h(t)}, \quad w(t, y)=u(t, h(t) y), \quad \phi_{i}(h(t) y)=\Phi_{i}(y), \quad i=1,2 .
$$

Then it follows from $(2.3)$ that $(w(t, y), h(t))$ satisfies

$$
\left\{\begin{array}{rlrl} 
& w_{t}-\zeta(t) w_{y y}-\xi(t) y w_{y} & \\
& \quad+\frac{\chi_{1} \mu_{1}}{\lambda_{1}} \zeta(t)\left(\Phi_{1} * w\right)_{y} w_{y}-\frac{\chi_{2} \mu_{2}}{\lambda_{2}} \zeta(t)\left(\Phi_{2} * w\right)_{y} w_{y} & \\
\quad & w\left[a-\left(b+\chi_{2} \mu_{2}-\chi_{1} \mu_{2}\right) w\right. & \\
\left.\quad-\chi_{1} \mu_{1}\left(\Phi_{1} * w\right)+\chi_{2} \mu_{2}\left(\Phi_{2} * w\right)\right], & t>0,0<y<1 \\
w_{y}(t, 0)=0, \quad w(t, y)=0, & t>0, y \geq 1 \\
w(0, y)=u_{0}\left(h_{0} y\right)=w_{0}(y), & 0 \leq y \leq 1,
\end{array}\right.
$$

and

$$
h^{\prime}(t)=-\mu \frac{1}{h(t)} w_{y}(t, 1), \quad t>0 ; \quad h(0)=h_{0},
$$

where $\zeta(t)=h^{-2}(t), \xi(t)=\frac{h^{\prime}(t)}{h(t)}$. Now problem (2.11) is an initial-boundary value problem with a fixed boundary. We shall use the fixed point theorem to prove the existence of solution $(w, h)$ to $(2.11)$ and $(2.12)$. Let $\tilde{h}=-\mu u_{0}^{\prime}\left(h_{0}\right), T_{1}=\min \left\{1, \frac{h_{0}}{2(2+\tilde{h})}\right\}$. For $0<T \leq T_{1}$, we set

$$
H_{T}=\left\{h \in C^{1}([0, T]): h(0)=h_{0}, h^{\prime}(0)=\tilde{h},\left\|h^{\prime}-\tilde{h}\right\|_{C([0, T])} \leq 1\right\}
$$

Clearly, $H_{T}$ is a bounded and closed convex set of $C^{1}([0, T])$. For the given $h \in H_{T}$, we can extend $h$ to a new function, still denoted by $h$, such that $h \in H_{T_{1}}$, where

$$
H_{T_{1}}=\left\{h \in C^{1}\left(\left[0, T_{1}\right]\right): h(0)=h_{0}, h^{\prime}(0)=\tilde{h},\left\|h^{\prime}-\tilde{h}\right\|_{C\left(\left[0, T_{1}\right]\right)} \leq 2\right\} .
$$

Therefore, when $h \in H_{T}$, we have $h \in H_{T_{1}}$ and

$$
\left|h(t)-h_{0}\right| \leq T_{1}\left\|h^{\prime}\right\|_{\infty} \leq T_{1}(2+\tilde{h}) \leq \frac{h_{0}}{2}, \quad \forall t \in\left[0, T_{1}\right]
$$

which yields

$$
\frac{h_{0}}{2} \leq h(t) \leq \frac{3 h_{0}}{2}, \quad \forall t \in\left[0, T_{1}\right]
$$


Thus, for the given $h \in H_{T}$, the functions $\zeta(t)$ and $\xi(t)$ are well defined on $\left[0, T_{1}\right]$. Let $R_{1} \triangleq \max \left\{\frac{a}{b+\chi_{2} \mu_{2}-\chi_{1} \mu_{1}-H},\left\|u_{0}\right\|_{L^{\infty}}\right\}$ where $H$ is given by (2.5). Define $\Delta_{T_{1}}=\left[0, T_{1}\right] \times[0,1]$ and

$$
W_{T_{1}}=\left\{w \in C\left(\Delta_{T_{1}}\right): w(0, y)=u_{0}\left(h_{0} y\right), w(t, 1)=0,0 \leq w(t, y) \leq R_{1}\right\} .
$$

Obviously, $W_{T_{1}}$ is a bounded and closed convex set of $C\left(\Delta_{T_{1}}\right)$. For the given $w \in W_{T_{1}}$, we have the following initial-boundary value problem

$$
\left\{\begin{array}{rlrl}
\bar{w}_{t}-\zeta(t) \bar{w}_{y y}-\xi(t) y \bar{w}_{y}+\frac{\chi_{1} \mu_{1}}{\lambda_{1}} \zeta(t)\left(\Phi_{1} * w\right)_{y} \bar{w}_{y} & \\
\quad-\frac{\chi_{2} \mu_{2}}{\lambda_{2}} \zeta(t)\left(\Phi_{2} * w\right)_{y} \bar{w}_{y} & \\
=\bar{w}\left[a-\left(b+\chi_{2} \mu_{2}-\chi_{1} \mu_{1}\right) \bar{w}\right. & \\
\left.\quad-\chi_{1} \mu_{1}\left(\Phi_{1} * w\right)+\chi_{2} \mu_{2}\left(\Phi_{2} * w\right)\right], & & 0<t \leq T_{1}, 0<y<1, \\
\bar{w}_{y}(t, 0)=0, \quad \bar{w}(t, y)=0, & & 0<t \leq T_{1}, y \geq 1, \\
\bar{w}(0, y)=w_{0}(y), & & 0 \leq y \leq 1 .
\end{array}\right.
$$

For the given $w \in W_{T_{1}}$, we obtain

$$
\begin{aligned}
\left(\Phi_{i} * w\right)(y):= & \int_{-1}^{0} \Phi_{i}(y-s) w(t,-s) d s \\
& +\int_{0}^{1} \Phi_{i}(y-s) w(t, s) d s, \quad y \in[0,1], i=1,2 .
\end{aligned}
$$

In order to prove that $0 \leq \bar{w}(t, y) \leq R_{1}$, we need the following estimate. Using (2.14), we have

$$
\begin{aligned}
\chi_{2} \mu_{2}\left(\Phi_{2} * w\right)-\chi_{1} \mu_{1}\left(\Phi_{1} * w\right) \\
=\chi_{2} \mu_{2}\left[\int_{-1}^{0} \frac{\sqrt{\lambda_{2}}}{2} e^{-\sqrt{\lambda_{2}}|y-s|} w(t,-s) d s+\int_{0}^{1} \frac{\sqrt{\lambda_{2}}}{2} e^{-\sqrt{\lambda_{2}}|y-s|} w(t, s) d s\right] \\
\quad-\chi_{1} \mu_{1}\left[\int_{-1}^{0} \frac{\sqrt{\lambda_{1}}}{2} e^{-\sqrt{\lambda_{1}}|y-s|} w(t,-s) d s+\int_{0}^{1} \frac{\sqrt{\lambda_{1}}}{2} e^{-\sqrt{\lambda_{1}}|y-s|} w(t, s) d s\right] \\
=\int_{-1}^{0}\left(\chi_{2} \mu_{2} \frac{\sqrt{\lambda_{2}}}{2} e^{-\sqrt{\lambda_{2}}|y-s|}-\chi_{1} \mu_{1} \frac{\sqrt{\lambda_{1}}}{2} e^{-\sqrt{\lambda_{1}}|y-s|}\right) w(t,-s) d s \\
\quad+\int_{0}^{1}\left(\chi_{2} \mu_{2} \frac{\sqrt{\lambda_{2}}}{2} e^{-\sqrt{\lambda_{2}}|y-s|}-\chi_{1} \mu_{1} \frac{\sqrt{\lambda_{1}}}{2} e^{-\sqrt{\lambda_{1}}|y-s|}\right) w(t, s) d s \\
=\left(\chi_{2} \lambda_{2} \mu_{2}-\chi_{1} \lambda_{1} \mu_{1}\right) \int_{-1}^{0} \frac{1}{2 \sqrt{\lambda_{2}}} e^{-\sqrt{\lambda_{2}}|y-s|} w(t,-s) d s \\
\quad+\chi_{1} \lambda_{1} \mu_{1} \int_{-1}^{0}\left(\frac{1}{2 \sqrt{\lambda_{2}}} e^{-\sqrt{\lambda_{2}}|y-s|}-\frac{1}{2 \sqrt{\lambda_{1}}} e^{-\sqrt{\lambda_{1}}|y-s|}\right) w(t,-s) d s \\
\quad+\left(\chi_{2} \lambda_{2} \mu_{2}-\chi_{1} \lambda_{1} \mu_{1}\right) \int_{0}^{1} \frac{1}{2 \sqrt{\lambda_{2}}} e^{-\sqrt{\lambda_{2}}|y-s|} w(t, s) d s \\
\quad+\chi_{1} \lambda_{1} \mu_{1} \int_{0}^{1}\left(\frac{1}{2 \sqrt{\lambda_{2}}} e^{-\sqrt{\lambda_{2}}|y-s|}-\frac{1}{2 \sqrt{\lambda_{1}}} e^{-\sqrt{\lambda_{1}}|y-s|}\right) w(t, s) d s
\end{aligned}
$$




$$
\begin{aligned}
\leq & \left(\chi_{2} \lambda_{2} \mu_{2}-\chi_{1} \lambda_{1} \mu_{1}\right)_{+} R_{1} \int_{-1}^{0} \frac{1}{2 \sqrt{\lambda_{2}}} e^{-\sqrt{\lambda_{2}}|y-s|} d s \\
& +\chi_{1} \lambda_{1} \mu_{1} R_{1} \int_{-1}^{0}\left(\frac{1}{2 \sqrt{\lambda_{2}}} e^{-\sqrt{\lambda_{2}}|y-s|}-\frac{1}{2 \sqrt{\lambda_{1}}} e^{-\sqrt{\lambda_{1}}|y-s|}\right) d s \\
& +\left(\chi_{2} \lambda_{2} \mu_{2}-\chi_{1} \lambda_{1} \mu_{1}\right)_{+} R_{1} \int_{0}^{1} \frac{1}{2 \sqrt{\lambda_{2}}} e^{-\sqrt{\lambda_{2}}|y-s|} d s \\
& +\chi_{1} \lambda_{1} \mu_{1} R_{1} \int_{0}^{1}\left(\frac{1}{2 \sqrt{\lambda_{2}}} e^{-\sqrt{\lambda_{2}}|y-s|}-\frac{1}{2 \sqrt{\lambda_{1}}} e^{-\sqrt{\lambda_{1}}|y-s|}\right) d s \\
= & \left(\chi_{2} \lambda_{2} \mu_{2}-\chi_{1} \lambda_{1} \mu_{1}\right)_{+} R_{1} \int_{-1}^{1} \frac{1}{2 \sqrt{\lambda_{2}}} e^{-\sqrt{\lambda_{2}}|y-s|} d s \\
& +\chi_{1} \lambda_{1} \mu_{1} R_{1} \int_{-1}^{1}\left(\frac{1}{2 \sqrt{\lambda_{2}}} e^{-\sqrt{\lambda_{2}}|y-s|}-\frac{1}{2 \sqrt{\lambda_{1}}} e^{-\sqrt{\lambda_{1}}|y-s|}\right) d s \\
\leq & \left(\chi_{2} \lambda_{2} \mu_{2}-\chi_{1} \lambda_{1} \mu_{1}\right)_{+} R_{1} \int_{\mathbb{R}} \frac{1}{2 \sqrt{\lambda_{2}}} e^{-\sqrt{\lambda_{2}}|y-s|} d s \\
& +\chi_{1} \lambda_{1} \mu_{1} R_{1} \int_{\mathbb{R}}\left(\frac{1}{2 \sqrt{\lambda_{2}}} e^{-\sqrt{\lambda_{2}}|y-s|}-\frac{1}{2 \sqrt{\lambda_{1}}} e^{-\sqrt{\lambda_{1}}|y-s|}\right) d s \\
= & \frac{R_{1}}{\lambda_{2}}\left[\left(\chi_{2} \lambda_{2} \mu_{2}-\chi_{1} \lambda_{1} \mu_{1}\right)_{+}+\chi_{1} \mu_{1}\left(\lambda_{1}-\lambda_{2}\right)_{+}\right] .
\end{aligned}
$$

Similarly, we have

$$
\begin{aligned}
& \chi_{2} \mu_{2}\left(\Phi_{2} * w\right)-\chi_{1} \mu_{1}\left(\Phi_{1} * w\right) \\
& =\chi_{2} \mu_{2}\left[\int_{-1}^{0} \frac{\sqrt{\lambda_{2}}}{2} e^{-\sqrt{\lambda_{2}}|y-s|} w(t,-s) d s+\int_{0}^{1} \frac{\sqrt{\lambda_{2}}}{2} e^{-\sqrt{\lambda_{2}}|y-s|} w(t, s) d s\right] \\
& -\chi_{1} \mu_{1}\left[\int_{-1}^{0} \frac{\sqrt{\lambda_{1}}}{2} e^{-\sqrt{\lambda_{1}}|y-s|} w(t,-s) d s+\int_{0}^{1} \frac{\sqrt{\lambda_{1}}}{2} e^{-\sqrt{\lambda_{1}}|y-s|} w(t, s) d s\right] \\
& =\int_{-1}^{0}\left(\chi_{2} \mu_{2} \frac{\sqrt{\lambda_{2}}}{2} e^{-\sqrt{\lambda_{2}}|y-s|}-\chi_{1} \mu_{1} \frac{\sqrt{\lambda_{1}}}{2} e^{-\sqrt{\lambda_{1}}|y-s|}\right) w(t,-s) d s \\
& +\int_{0}^{1}\left(\chi_{2} \mu_{2} \frac{\sqrt{\lambda_{2}}}{2} e^{-\sqrt{\lambda_{2}}|y-s|}-\chi_{1} \mu_{1} \frac{\sqrt{\lambda_{1}}}{2} e^{-\sqrt{\lambda_{1}}|y-s|}\right) w(t, s) d s \\
& =\left(\chi_{2} \lambda_{2} \mu_{2}-\chi_{1} \lambda_{1} \mu_{1}\right) \int_{-1}^{0} \frac{1}{2 \sqrt{\lambda_{1}}} e^{-\sqrt{\lambda_{1}}|y-s|} w(t,-s) d s \\
& +\chi_{2} \lambda_{2} \mu_{2} \int_{-1}^{0}\left(\frac{1}{2 \sqrt{\lambda_{2}}} e^{-\sqrt{\lambda_{2}}|y-s|}-\frac{1}{2 \sqrt{\lambda_{1}}} e^{-\sqrt{\lambda_{1}}|y-s|}\right) w(t,-s) d s \\
& +\left(\chi_{2} \lambda_{2} \mu_{2}-\chi_{1} \lambda_{1} \mu_{1}\right) \int_{0}^{1} \frac{1}{2 \sqrt{\lambda_{1}}} e^{-\sqrt{\lambda_{1}}|y-s|} w(t, s) d s \\
& +\chi_{2} \lambda_{2} \mu_{2} \int_{0}^{1}\left(\frac{1}{2 \sqrt{\lambda_{2}}} e^{-\sqrt{\lambda_{2}}|y-s|}-\frac{1}{2 \sqrt{\lambda_{1}}} e^{-\sqrt{\lambda_{1}}|y-s|}\right) w(t, s) d s \\
& \leq\left(\chi_{2} \lambda_{2} \mu_{2}-\chi_{1} \lambda_{1} \mu_{1}\right)_{+} R_{1} \int_{-1}^{0} \frac{1}{2 \sqrt{\lambda_{1}}} e^{-\sqrt{\lambda_{1}}|y-s|} d s \\
& +\chi_{2} \lambda_{2} \mu_{2} R_{1} \int_{-1}^{0}\left(\frac{1}{2 \sqrt{\lambda_{2}}} e^{-\sqrt{\lambda_{2}}|y-s|}-\frac{1}{2 \sqrt{\lambda_{1}}} e^{-\sqrt{\lambda_{1}}|y-s|}\right) d s
\end{aligned}
$$




$$
\begin{aligned}
& +\left(\chi_{2} \lambda_{2} \mu_{2}-\chi_{1} \lambda_{1} \mu_{1}\right)_{+} R_{1} \int_{0}^{1} \frac{1}{2 \sqrt{\lambda_{1}}} e^{-\sqrt{\lambda_{1}}|y-s|} d s \\
& +\chi_{2} \lambda_{2} \mu_{2} R_{1} \int_{0}^{1}\left(\frac{1}{2 \sqrt{\lambda_{2}}} e^{-\sqrt{\lambda_{2}}|y-s|}-\frac{1}{2 \sqrt{\lambda_{1}}} e^{-\sqrt{\lambda_{1}}|y-s|}\right) d s \\
= & \left(\chi_{2} \lambda_{2} \mu_{2}-\chi_{1} \lambda_{1} \mu_{1}\right)_{+} R_{1} \int_{-1}^{1} \frac{1}{2 \sqrt{\lambda_{1}}} e^{-\sqrt{\lambda_{1}}|y-s|} d s \\
& +\chi_{2} \lambda_{2} \mu_{2} R_{1} \int_{-1}^{1}\left(\frac{1}{2 \sqrt{\lambda_{2}}} e^{-\sqrt{\lambda_{2}}|y-s|}-\frac{1}{2 \sqrt{\lambda_{1}}} e^{-\sqrt{\lambda_{1}}|y-s|}\right) d s \\
\leq & \left(\chi_{2} \lambda_{2} \mu_{2}-\chi_{1} \lambda_{1} \mu_{1}\right)_{+} R_{1} \int_{\mathbb{R}} \frac{1}{2 \sqrt{\lambda_{1}}} e^{-\sqrt{\lambda_{1}}|y-s|} d s \\
& +\chi_{2} \lambda_{2} \mu_{2} R_{1} \int_{\mathbb{R}}\left(\frac{1}{2 \sqrt{\lambda_{2}}} e^{-\sqrt{\lambda_{2}}|y-s|}-\frac{1}{2 \sqrt{\lambda_{1}}} e^{-\sqrt{\lambda_{1}}|y-s|}\right) d s \\
= & \frac{R_{1}}{\lambda_{1}}\left[\left(\chi_{2} \lambda_{2} \mu_{2}-\chi_{1} \lambda_{1} \mu_{1}\right)_{+}+\chi_{2} \mu_{2}\left(\lambda_{1}-\lambda_{2}\right)_{+}\right] .
\end{aligned}
$$

Thus, it follows from (2.15) and (2.16) that for every $w \in W_{T_{1}}$, we have

$$
\chi_{2} \mu_{2}\left(\Phi_{2} * w\right)-\chi_{1} \mu_{1}\left(\Phi_{1} * w\right) \leq H R_{1}
$$

where $H$ is given by (2.5). Thus in view of (2.13) and (2.17), we have

$$
\begin{aligned}
& \bar{w}_{t}-\zeta(t) \bar{w}_{y y}-\xi(t) y \bar{w}_{y}+\frac{\chi_{1} \mu_{1}}{\lambda_{1}} \zeta(t)\left(\Phi_{1} * w\right)_{y} \bar{w}_{y}-\frac{\chi_{2} \mu_{2}}{\lambda_{2}} \zeta(t)\left(\Phi_{2} * w\right)_{y} \bar{w}_{y} \\
& \quad \leq \bar{w}\left[a+H R_{1}-\left(\chi_{2} \mu_{2}+b-\chi_{1} \mu_{1}\right) \bar{w}\right] .
\end{aligned}
$$

Note that

$$
L\left(R_{1}\right)=R_{1}\left[a-\left(b+\chi_{2} \mu_{2}-\chi_{1} \mu_{1}-H\right) R_{1}\right] \leq 0 .
$$

Hence, using the comparison principle for parabolic equations, we obtain that

$$
\bar{w}(t, y) \leq R_{1}, \quad t \in\left(0, T_{1}\right], y \in[0,1], \forall w \in W_{T_{1}} .
$$

Applying the maximum principle, we infer that $\bar{w}>0$ in $\left(0, T_{1}\right] \times[0,1)$. Hence $\bar{w}$ is a bounded function. Straightforward calculation yields

$$
\begin{aligned}
\left(\Phi_{i} * w\right)_{y} & \\
= & \int_{-\infty}^{0}-\frac{\lambda_{i}}{2} e^{-\sqrt{\lambda_{i}}(y-s)} w(t,-s) d s+\int_{0}^{y}-\frac{\lambda_{i}}{2} e^{-\sqrt{\lambda_{i}}(y-s)} w(t, s) d s \\
& +\int_{y}^{+\infty} \frac{\lambda_{i}}{2} e^{-\sqrt{\lambda_{i}}(s-y)} w(t, s) d s \\
= & \sqrt{\lambda_{i}}\left[\int_{-\infty}^{0}-\frac{\sqrt{\lambda_{i}}}{2} e^{-\sqrt{\lambda_{i}}(y-s)} w(t,-s) d s+\int_{0}^{y}-\frac{\sqrt{\lambda_{i}}}{2} e^{-\sqrt{\lambda_{i}}(y-s)} w(t, s) d s\right. \\
& \left.+\int_{y}^{+\infty} \frac{\sqrt{\lambda_{i}}}{2} e^{-\sqrt{\lambda_{i}}(s-y)} w(t, s) d s\right] .
\end{aligned}
$$


Thus,

$$
\left|\left(\Phi_{i} * w\right)_{y}\right| \leq \sqrt{\lambda_{i}}\left(\Phi_{i} * w\right) \leq \sqrt{\lambda_{i}}\|w\|_{L^{\infty}} \leq \sqrt{\lambda_{i}} R_{1} .
$$

By the standard method (the upper and lower solutions method, or the contraction mapping theory, for example), we can show that there exists $0<T_{*} \leq T_{1}$, depending only on $\Lambda=\left\{R_{1}, \chi_{i}, \mu_{i}, \lambda_{i}, h_{0}, \tilde{h},\left\|u_{0}\right\|_{W^{2, p}\left(\left[0, h_{0}\right]\right)}, i=1,2\right\}$, such that problem (2.13) admits a unique solution $\bar{w} \in W_{p}^{1,2}\left(\Delta_{T_{*}}\right) \hookrightarrow C^{(1+\theta) / 2,1+\theta}\left(\Delta_{T_{*}}\right)$, which satisfies

$$
\|\bar{w}\|_{W_{p}^{1,2}\left(\Delta_{T_{*}}\right)}+\|\bar{w}\|_{C^{(1+\theta) / 2,1+\theta}\left(\Delta_{T_{*}}\right)} \leq C_{1}\left(\Lambda, T_{*}, T_{*}^{-1}\right)
$$

where

$$
\Delta_{T_{*}}=\left[0, T_{*}\right] \times[0,1] .
$$

It follows from (2.18) and (2.19) that the bound of the right-hand side of the first equation in (2.13) depends only on $\Lambda$. We may think that $T_{*}$ depends only on $\Lambda$ and write $C_{1}\left(\Lambda, T_{*}, T_{*}^{-1}\right)$ as $C_{1}(\Lambda)$. That is,

$$
\|\bar{w}\|_{W_{p}^{1,2}\left(\Delta_{T_{*}}\right)}+\|\bar{w}\|_{C^{(1+\theta) / 2,1+\theta}\left(\Delta_{T_{*}}\right)} \leq C_{1}(\Lambda) .
$$

Therefore, when $0<T \leq T^{*}$, the unique solution $\bar{w}(t, y)$ of $(2.13)$ satisfies

$$
\|\bar{w}\|_{W_{p}^{1,2}\left(\Delta_{T}\right)}+\|\bar{w}\|_{C^{(1+\theta) / 2,1+\theta}\left(\Delta_{T}\right)} \leq\|\bar{w}\|_{W_{p}^{1,2}\left(\Delta_{T_{*}}\right)}+\|\bar{w}\|_{C^{(1+\theta) / 2,1+\theta}\left(\Delta_{T_{*}}\right)} \leq C_{1}(\Lambda) .
$$

We now define $\digamma: W_{T} \rightarrow C\left(\Delta_{T}\right)$ by

$$
\digamma(w)=\bar{w} .
$$

It follows from (2.18) and (2.20) that $\digamma$ maps $W_{T}$ into itself.

Next, we prove that $\digamma$ is a contraction mapping on $W_{T}$ for $T>0$ sufficiently small. Indeed, let $w_{i} \in W_{T}(\mathrm{i}=1,2)$ and denote $\bar{w}_{i}=\digamma\left(w_{i}\right)$. Then it follows from (2.21) that

$$
\left\|\bar{w}_{i}\right\|_{W_{p}^{2,1}\left(\Delta_{T}\right)} \leq C_{1}(\Lambda)
$$

Setting $U=\bar{w}_{1}-\bar{w}_{2}$, we find that $U(t, y)$ satisfies

$$
\begin{cases}U_{t}-\zeta(t) U_{y y}-\xi(t) y U_{y}-\alpha U & \\ \quad+\left[\frac{\chi_{1} \mu_{1}}{\lambda_{1}} \zeta(t)\left(\Phi_{1} * w_{1}\right)_{y}-\frac{\chi_{2} \mu_{2}}{\lambda_{2}} \zeta(t)\left(\Phi_{2} * w_{1}\right)_{y}\right] U_{y}=\beta, & 0<t \leq T, 0<y<1, \\ U_{y}(t, 0)=0, \quad U(t, y)=0, & 0<t \leq T, y \geq 1, \\ U(0, y)=0, & 0 \leq y \leq 1,\end{cases}
$$

where $\zeta(t)=\zeta(h(t))$ and $\xi(t)=\xi\left(h(t), h^{\prime}(t)\right)$,

$$
\alpha=a-\left(b+\chi_{2} \mu_{2}-\chi_{1} \mu_{1}\right)\left(\bar{w}_{1}+\bar{w}_{2}\right)-\chi_{1} \mu_{1}\left(\Phi_{1} * w_{2}\right)+\chi_{2} \mu_{2}\left(\Phi_{2} * w_{2}\right),
$$




$$
\begin{aligned}
\beta= & {\left[\frac{\chi_{1} \mu_{1}}{\lambda_{1}} \zeta(t)\left(\Phi_{1} *\left(w_{1}-w_{2}\right)\right)_{y}-\frac{\chi_{2} \mu_{2}}{\lambda_{2}} \zeta(t)\left(\Phi_{2} *\left(w_{1}-w_{2}\right)\right)_{y}\right] \bar{w}_{2, y} } \\
& +\bar{w}_{1}\left[\chi_{1} \mu_{1}\left(\Phi_{1} *\left(w_{2}-w_{1}\right)\right)+\chi_{2} \mu_{2}\left(\Phi_{2} *\left(w_{1}-w_{2}\right)\right)\right] .
\end{aligned}
$$

Applying the classical $L^{p}$ estimates for parabolic equations and (2.19), we obtain

$$
\left\|\bar{w}_{1}-\bar{w}_{2}\right\|_{W_{p}^{2,1}\left(\Delta_{T}\right)} \leq C_{2}(T, \Lambda)\left\|w_{1}-w_{2}\right\|_{L^{p}\left(\Delta_{T}\right)}
$$

Using Hölder's inequality and (2.22), we have

$$
\begin{aligned}
\left\|\bar{w}_{1}-\bar{w}_{2}\right\|_{L^{p}\left(\Delta_{T}\right)}^{p} & =\int_{0}^{T} \int_{0}^{1}\left|\bar{w}_{1}-\bar{w}_{2}\right|^{p} d y d t \\
& =\int_{0}^{T} \int_{0}^{1}\left|\int_{0}^{t}\left[\bar{w}_{1}(\tau, y)-\bar{w}_{2}(\tau, y)\right]_{\tau} d \tau\right|^{p} d y d t \\
& \leq \int_{0}^{T} \int_{0}^{1}\left(\int_{0}^{t}\left[\bar{w}_{1}(\tau, y)-\bar{w}_{2}(\tau, y)\right]_{\tau}^{p} d \tau\right) t^{p-1} d y d t \\
& \leq T^{p-1} \int_{0}^{T} \int_{0}^{1} \int_{0}^{T}\left[\bar{w}_{1}(\tau, y)-\bar{w}_{2}(\tau, y)\right]_{\tau}^{p} d \tau d y d t \\
& \leq T^{p}\left\|\left(\bar{w}_{1}-\bar{w}_{2}\right)_{t}\right\|_{L^{p}\left(\Delta_{T}\right)}^{p} \\
& \leq T^{p}\left\|\bar{w}_{1}-\bar{w}_{2}\right\|_{W_{p}^{2,1}\left(\Delta_{T}\right)}^{p} \\
& \leq C_{2}(T, \Lambda) T^{p}\left\|w_{1}-w_{2}\right\|_{L_{p}\left(\Delta_{T}\right)}^{p},
\end{aligned}
$$

where $C_{2}$ is a constant independent on $T^{-1}$. We choose $T$ small enough such that $C_{2} T^{p}<1$. This shows that for this $T, \digamma$ is a contraction mapping on $W_{T}$. It now follows from the contraction mapping theorem that $\digamma$ has a unique fixed point $w \in W_{T}$. Therefore, (2.11) has a unique solution $w(t, y)$.

By the continuous dependence on the given data, in the space $C^{(1+\theta) / 2,1+\theta}\left(\Delta_{T}\right), w$ depends continuously on $h \in H_{T}$. For such a defined function $w$, the initial value problem (2.12) has a unique solution, denoted by $\bar{h}(t)=\bar{h}(t ; h)$. Then $\bar{h}(0)=h_{0}, \bar{h}^{\prime}(0)=\tilde{h}$ and

$$
\bar{h}^{\prime}(t)>0, \quad \bar{h}^{\prime}(t) \in C^{\theta / 2}([0, T]), \quad\left\|\bar{h}^{\prime}\right\|_{C^{\theta / 2}([0, T])} \leq C_{3}(\Lambda), \quad \forall h \in H_{T} .
$$

Clearly, in the space $C^{1}([0, T]), \bar{h}$ continuously depends on $w \in C^{(1+\theta) / 2,1+\theta}\left(\Delta_{T}\right)$, and hence on $h \in H_{T}$. Now we define $\mathcal{F}: H_{T} \rightarrow C^{1}([0, T])$ by

$$
\mathcal{F}(h)=\bar{h} \text {. }
$$

Obviously, $\mathcal{F}$ is continuous in $H_{T}$, and $h \in H_{T}$ is a fixed point of $\mathcal{F}$ if and only if ( $\left.w, h\right)$ solves (2.11) and (2.12).

According to (2.23), we know that $\mathcal{F}$ is compact and

$$
\left\|\bar{h}^{\prime}-\tilde{h}\right\|_{C([0, T])} \leq\left\|\bar{h}^{\prime}\right\|_{C^{\theta / 2([0, T])}} T^{\theta / 2} \leq C_{3}(\Lambda) T^{\theta / 2} .
$$


Hence $\mathcal{F}$ maps $H_{T}$ into itself if

$$
0<T \leq \min \left\{1, \frac{h_{0}}{2(2+\tilde{h})}, C_{3}^{-2 / \theta}(\Lambda)\right\}
$$

Consequently, $\mathcal{F}$ has at least one fixed point $h \in H_{T}$ by Schauder's fixed point theorem, and then (2.11) and (2.12) have at least one solution $(w, h)$ defined in $[0, T]$. Moreover, as $w(t, y)>0$ and $w(t, 1)=0$, we deduce by Hopf's boundary lemma that $w_{y}(t, 1)<0$, which implies $h^{\prime}(t)>0$ for $t>0$.

Obviously, the function $u(t, x)=w\left(t, h^{-1} x\right)$ satisfies

$$
u \in W_{p}^{1,2}\left(D_{T}\right) \cap C^{(1+\theta) / 2,1+\theta}\left(\bar{D}_{T}\right), \quad 0<u \leq R_{1} \text { in } D_{T}
$$

$(u, h)$ solves $(1.1)$ and

$$
h \in C^{1+\theta / 2}([0, T]) .
$$

Let $\left(w_{i}, h_{i}\right)$ with $i=1,2$, be two local solutions of (2.11), which are defined for $t \in[0, T]$ and $0<T \ll 1$. Notice $h_{i}^{\prime}(t)>0$. We may assume that $h_{0} \leq h_{i}(t) \leq h_{0}+1$ in $[0, T], i=$ 1,2 .

Setting $\mathcal{U}=w_{1}-w_{2}$ and $h=h_{1}-h_{2}$, we have

$$
\begin{cases}\mathcal{U}_{t}-\zeta_{2}(t) \mathcal{U}_{y y}-\xi_{2}(t) y \mathcal{U}_{y}-A \mathcal{U} & \\ \quad+w_{1}\left[\chi _ { 2 } \mu _ { 2 } \left(\left(\Phi_{2} * \mathcal{U}\right)-\chi_{1} \mu_{1}\left(\left(\Phi_{1} * \mathcal{U}\right)\right]\right.\right. & \\ \quad+\left[\frac{\chi_{1} \mu_{1}}{\lambda_{1}} \zeta_{2}(t)\left(\Phi_{1} * w_{2}\right)_{y}-\frac{\chi_{2} \mu_{2}}{\lambda_{2}} \zeta_{2}(t)\left(\Phi_{2} * w_{2}\right)_{y}\right] \mathcal{U}_{y}=B, & 0<t \leq T, 0<y<1, \\ \mathcal{U}_{y}(t, 0)=0, \quad \mathcal{U}(t, y)=0, & 0<t \leq T, y \geq 1 \\ \mathcal{U}(0, y)=0, & 0 \leq y \leq 1,\end{cases}
$$

and

$$
h^{\prime}(t)=\mu\left(\frac{1}{h_{2}} w_{2, y}(t, 1)-\frac{1}{h_{1}} w_{1, y}(t, 1)\right), \quad 0<t \leq T ; \quad h(0)=0,
$$

where $\zeta_{i}(t)=\zeta\left(h_{i}(t)\right)$ and $\xi_{i}(t)=\xi\left(h_{i}(t), h_{i}^{\prime}(t)\right), i=1,2$,

$$
\begin{aligned}
A= & a-\left(b+\chi_{2} \mu_{2}-\chi_{1} \mu_{1}\right)\left(w_{1}+w_{2}\right)-\chi_{1} \mu_{1}\left(\Phi_{1} * w_{2}\right)+\chi_{2} \mu_{2}\left(\Phi_{2} * w_{2}\right), \\
B= & \left(\zeta_{1}(t)-\zeta_{2}(t)\right) w_{1, y y}+\left(\xi_{1}(t)-\xi_{2}(t)\right) y w_{1, y} \\
& +\left[\zeta_{1}(t)\left(\frac{\chi_{1} \mu_{1}}{\lambda_{1}}\left(\Phi_{1} * w_{1}\right)_{y}-\frac{\chi_{2} \mu_{2}}{\lambda_{2}}\left(\Phi_{2} * w_{1}\right)_{y}\right)\right. \\
& \left.-\zeta_{2}(t)\left(\frac{\chi_{1} \mu_{1}}{\lambda_{1}}\left(\Phi_{1} * w_{2}\right)_{y}-\frac{\chi_{2} \mu_{2}}{\lambda_{2}}\left(\Phi_{2} * w_{2}\right)_{y}\right)\right] w_{1, y} .
\end{aligned}
$$


By (2.19), $\left\|w_{1}\right\|_{W_{p}^{1,2}} \leq C_{1}(\Lambda), 0<h_{i}^{\prime}(t) \leq C_{3}(\Lambda)$ and $h_{0} \leq h_{i}(t) \leq h_{0}+1$. Applying the classical $L^{p}$ estimates for parabolic equations, we obtain

$$
\begin{aligned}
\|\mathcal{U}\|_{W_{p}^{1,2}\left(\Delta_{T}\right)} \leq & C_{4}(\Lambda)\left(\left\|\left(h_{1}^{-2}-h_{2}^{-2}\right) w_{1, y y}\right\|_{L^{p}\left(\Delta_{T}\right)}+\left\|\left(h_{1}^{\prime} / h_{1}-h_{2}^{\prime} / h_{2}\right) y w_{1, y}\right\|_{L^{p}\left(\Delta_{T}\right)}\right. \\
& \left.+\left\|\left(h_{1}^{-2}-h_{2}^{-2}\right) w_{1, y}\right\|_{L^{p}\left(\Delta_{T}\right)}\right) \\
\leq & C_{5}(\Lambda)\|h\|_{C^{1}([0, T])} .
\end{aligned}
$$

By similar arguments as in the proof of Theorem 1.1 of [36], we can obtain that

$$
[\mathcal{U}]_{C^{\theta / 2, \theta}\left(\Delta_{T}\right)},\left[\mathcal{U}_{y}\right]_{C^{\theta / 2, \theta}\left(\Delta_{T}\right)} \leq C\|\mathcal{U}\|_{W_{p}^{1,2}\left(\Delta_{T}\right)}
$$

for some positive constant $C$ independent of $T^{-1}$, here $[\cdot]_{C^{\theta / 2, \theta}\left(\Delta_{T}\right)}$ is a Hölder seminorm. Thus,

$$
\left[\mathcal{U}_{y}\right]_{C^{\theta / 2, \theta}\left(\Delta_{T}\right)} \leq C C_{5}(\Lambda)\|h\|_{C^{1}([0, T])} .
$$

This, combined with (2.25), yields

$$
\begin{aligned}
{\left[h^{\prime}\right]_{C^{\theta / 2}([0, T])} } & \leq \mu\left[h_{1}^{-1} \mathcal{U}_{y}(t, 1)\right]_{C^{\theta / 2}([0, T])}+\mu\left[\left(h_{1}^{-1}-h_{2}^{-1}\right) w_{2, y}(t, 1)\right]_{C^{\theta / 2}([0, T])} \\
& \leq C_{6}(\Lambda)\|h\|_{C^{1}([0, T])} .
\end{aligned}
$$

Because of $h(0)=h^{\prime}(0)=0$, we deduce

$$
\|h\|_{C^{1}([0, T])} \leq 2 T^{\theta / 2}\left\|h^{\prime}\right\|_{C^{\theta / 2([0, T])}} \leq 2 C_{6}(\Lambda) T^{\theta / 2}\|h\|_{C^{1}([0, T])} .
$$

We can choose $0<\hat{T}(\Lambda) \ll 1$ such that, when $0<T \leq \hat{T}(\Lambda), h_{1}=h_{2}$, and consequently $w_{1}=w_{2}$. Hence, (1.1) has a unique local classical solution $\left(u, v_{1}, v_{2}, h\right)$, i.e., (2.7) holds.

Let $0<\tau<\infty$ and $\left(u, v_{1}, v_{2}, h\right) \in W_{p}^{1,2}\left(D_{T}\right) \cap C^{(1+\theta) / 2,1+\theta}\left(\bar{D}_{T}\right) \times\left[C^{(1+\theta) / 2,1+\theta}\left(D_{v}^{T}\right)\right]^{2} \times$ $C^{1+\theta / 2}([0, T])$ be the unique solution of (1.1). It follows from the maximum principle that $u, v_{i}>0, i=1,2$ in $[0, \tau] \times[0, h(t))$. Thus, $u_{x}(t, h(t))<0$ for $t \in(0, \tau]$, and we see from (1.1) that $h^{\prime}(t)>0$ in $(0, \tau]$.

According to (2.24), we have

$$
0<u(t, x) \leq \max \left\{\frac{a}{b+\chi_{2} \mu_{2}-\chi_{1} \mu_{1}-H},\left\|u_{0}\right\|_{L^{\infty}}\right\}=R_{1}, \quad(t, x) \in(0, \tau) \times[0, h(t)) .
$$

Applying the maximum principle to the second and third equations in (1.1), we obtain

$$
0<v_{i}(t, x) \leq \max \left\{\frac{\mu_{i}}{\lambda_{i}} \frac{a}{b+\chi_{2} \mu_{2}-\chi_{1} \mu_{1}-H}, \frac{\mu_{i}}{\lambda_{i}}\left\|u_{0}\right\|_{L^{\infty}}\right\}, \quad(t, x) \in(0, \tau) \times[0,+\infty) .
$$

As we all know, the proof of (2.10) is standard. The proof is the same as that of Lemma 2.2 in [7], thus we omit the details.

Similar to the proofs of Theorem 2.1, Lemma 2.2 and Theorem 2.3 in [7], we can obtain the following global existence result. 
Theorem 2.2 Suppose that (2.4) holds. For all $t \in(0, \infty)$, problem (1.1) possesses a unique solution $\left(u, v_{1}, v_{2}, h\right)$, and for any $\theta \in(0,1)$,

$$
\begin{aligned}
& u \in C^{1+\theta / 2,2+\theta}((0, \infty) \times(0, h(t))), \quad h \in C^{1+(1+\theta) / 2}((0, \infty)), \\
& v_{i} \in C^{1+\theta / 2,2+\theta}((0, \infty) \times(0, \infty)), \quad i=1,2 .
\end{aligned}
$$

Moreover, there exists some constant $C$ dependent on $\chi_{i}, \mu_{i}, \lambda_{i}, a, b, h_{0}$ and $\left\|u_{0}\right\|_{\infty}$ such that

$$
\begin{aligned}
& \|u(t, \cdot)\|_{C^{1}([0, h(t)])} \leq C, \quad\left\|v_{i}(t, \cdot)\right\|_{C^{1}([0, \infty))} \leq C, \quad \forall t \geq 1, i=1,2, \\
& \left\|h^{\prime}\right\|_{C^{\frac{\theta}{2}([1,+\infty))}} \leq C, \quad \forall n \geq 0 .
\end{aligned}
$$

The proof of Theorem 2.2 in essence follows similar arguments as in [37-39, 42]. For the regularity results and estimates of $\left(u, v_{1}, v_{2}, h\right)$, please refer to Theorem 2.2 of [39]. The details are omitted here. We remark that the uniform estimate (2.30) allows us to deduce that $h^{\prime}(t) \rightarrow 0$ when $\lim _{t \rightarrow \infty} h(t)<\infty$, which plays a key role in determining the vanishing phenomenon.

\section{Long time behavior of $(u, v)$}

This section is devoted to proving the asymptotic behavior when $t \rightarrow \infty$. It follows from Lemma 2.1 that $h(t)$ is monotonically increasing. Therefore, there exists an $h_{\infty} \in(0, \infty]$ such that $\lim _{t \rightarrow \infty} h(t)=h_{\infty}$.

\subsection{Vanishing case $\left(h_{\infty}<\infty\right)$}

In this subsection, we study the asymptotic behavior of solution to problem (1.1) when vanishing occurs $\left(h_{\infty}<\infty\right)$. Our approaches here are mainly following the lines of [32, 41]. First, we state a general result.

Theorem 3.1 Suppose that (2.4) holds. Let $\left(u, v_{1}, v_{2}, h\right)$ be the solution of (1.1). If $h_{\infty}<\infty$, then there exists a constant $C>0$ such that

$$
\|u(t, \cdot)\|_{C^{1}([0, h(t)])} \leq C, \quad \forall t>1 .
$$

Moreover,

$$
\lim _{t \rightarrow+\infty} h^{\prime}(t)=0
$$

Proof The proof is similar to that of Theorem 4.1 in [41]. We omit the details.

Similar to Proposition 3.2 in [52], we can prove the following general proposition.

Proposition 3.2 Let $\mu>0, c \in \mathbb{R}$. Assume that $\in C^{1}([0, \infty))$ and $w \in C^{(1+v) / 2,(1+v)}([0, \infty) \times$ $[0, s(t)])$ satisfy $s(t)>0, w(t, x)>0$, for all $t \geq 0,0<x<s(t)$. We further suppose that $s_{\infty}=$ $\lim _{t \rightarrow \infty} s(t)<\infty, \lim _{t \rightarrow \infty} s^{\prime}(t)=0$ and there exists a constant $C$ such that $\|w(t, \cdot)\|_{C^{1}[0, s(t)]} \leq$ 
C for $t>1$. If $(w, s)$ satisfies

$$
\begin{cases}w_{t}-w_{x x}+m_{x} w_{x} \geq c w, & t>0,0<x<s(t), \\ w_{x}(t, 0)=0, & t>0, \\ w(t, s(t))=0, \quad s^{\prime}(t) \geq-\mu w_{x}(t, s(t)), & t \geq 0,\end{cases}
$$

then $\lim _{t \rightarrow \infty} \max _{0 \leq x \leq s(t)} w(t, x)=0$.

Theorem 3.3 Suppose that (2.4) holds. Let $\left(u, v_{1}, v_{2}, h\right)$ be any solution of problem (1.1). If $h_{\infty}<\infty$, then

$$
\begin{aligned}
& \lim _{t \rightarrow \infty}\|u(t, \cdot)\|_{C([0, h(t)])}=0, \\
& \lim _{t \rightarrow \infty} v_{i}(t, x)=0, \quad i=1,2 \text { uniformly in }[0,+\infty) .
\end{aligned}
$$

Proof First, we can easily obtain that $u$ satisfies

$$
\begin{cases}u_{t}-u_{x x}+\left(\chi_{1} v_{1, x}-\chi_{2} v_{2, x}\right) u_{x} \geq c u, & t>0,0<x<h(t) \\ u_{x}(t, 0)=0, \quad u(t, h(t))=0, & t \geq 0, \\ u(0, x)=u_{0}(x), & 0 \leq x \leq h_{0}\end{cases}
$$

where $c \leq \min _{x \in[0, \infty)}\left[a-\left(b+\chi_{2} \mu_{2}-\chi_{1} \mu_{1}\right) u-\chi_{1} \lambda_{1} v_{1}\right]$. Note that $h^{\prime}(t)>0$ and $h_{\infty}<\infty$, in view of Theorem 3.1 we get $\lim _{t \rightarrow \infty} h^{\prime}(t)=0$. Using Proposition 3.2, we have

$$
\lim _{t \rightarrow \infty}\|u(t, \cdot)\|_{C([0, h(t)])}=0
$$

Combining (2.1), (2.2) and $\lim _{t \rightarrow \infty}\|u(t, \cdot)\|_{C([0, h(t)])}=0$, we deduce

$$
\lim _{t \rightarrow \infty} v_{i}(t, x)=0, \quad i=1,2 \text {, uniformly in }[0,+\infty) .
$$

The proof is complete.

\subsection{Spreading case $\left(h_{\infty}=\infty\right)$}

In this subsection, we discuss the spreading case $h_{\infty}=\infty$. We provide a sufficient condition (1.3) to prove that system (1.1) has a unique positive equilibrium $\left(a / b, \mu_{1} a / \lambda_{1} b\right.$, $\left.\mu_{2} a / \lambda_{2} b\right)$. This condition is more stringent than (2.4). The proof is inspired by Theorem 4.3 in [41].

Next, to discuss the asymptotic behavior of the solution to problem (1.1), we intend to control the bounds of $v_{1}$ and $v_{2}$ by using those of $u$. Thus, we provide two lemmas, which will be used in the main theorem in this subsection.

Lemma 3.4 Let $\left(u, v_{1}, v_{2}, h\right)$ be a solution of problem (1.1). If (2.4) holds and $u$ satisfies

$$
\limsup _{t \rightarrow \infty} u(t, x) \leq \bar{A} \quad \text { uniformly in any bounded subset of }[0,+\infty)
$$


Chang et al. Boundary Value Problems

(2018) 2018:191

Page 15 of 38

with some constant $\bar{A}>0$, then we have

$$
\limsup _{t \rightarrow \infty} v_{i}(t, x) \leq \frac{\mu_{i}}{\lambda_{i}} \bar{A}, \quad i=1,2, \text { uniformly in any bounded subset of }[0,+\infty) \text {. }
$$

Proof For any given $L>0$ and $0<\varepsilon \ll 1$, we can choose a small positive constant $\delta$ and $n>0$ large enough such that

$$
\frac{\mu_{i}}{\lambda_{i}} \delta+\frac{\mu_{i} R_{1}}{2 \sqrt{\lambda_{i}}} e^{-\sqrt{\lambda_{i}} n L}\left(1+e^{\sqrt{\lambda_{i} L}}\right)<\varepsilon
$$

where $R_{1}$ is defined in Lemma 2.1. For $n L>0$ and $0<\delta \ll 1$, in view of (3.7), there exists a $T>0$ such that

$$
u(t, x) \leq \bar{A}+\delta, \quad \text { for all } t>T \text { and } x \in[0, n L]
$$

According to (3.8), (3.9), direct calculations show that

$$
\begin{aligned}
& v_{i}(t, x) \\
& =\frac{\mu_{i}}{2 \sqrt{\lambda_{i}}}\left[\int_{-\infty}^{0} e^{-\sqrt{\lambda_{i}}|x-y|} u(t,-y) d y+\int_{0}^{+\infty} e^{-\sqrt{\lambda_{i}}|x-y|} u(t, y) d y\right] \\
& =\frac{\mu_{i}}{2 \sqrt{\lambda_{i}}}\left[\int_{-\infty}^{0} e^{-\sqrt{\lambda_{i}}(x-y)} u(t,-y) d y+\int_{0}^{x} e^{-\sqrt{\lambda_{i}}(x-y)} u(t, y) d y\right] \\
& +\frac{\mu_{i}}{2 \sqrt{\lambda_{i}}}\left[\int_{x}^{n L} e^{-\sqrt{\lambda_{i}}(y-x)} u(t, y) d y+\int_{n L}^{+\infty} e^{-\sqrt{\lambda_{i}}(y-x)} u(t, y) d y\right] \\
& =\frac{\mu_{i}}{2 \sqrt{\lambda_{i}}}\left[\int_{0}^{+\infty} e^{-\sqrt{\lambda_{i}}(x+y)} u(t, y) d y+\int_{0}^{x} e^{-\sqrt{\lambda_{i}}(x-y)} u(t, y) d y\right] \\
& +\frac{\mu_{i}}{2 \sqrt{\lambda_{i}}}\left[\int_{x}^{n L} e^{-\sqrt{\lambda_{i}}(y-x)} u(t, y) d y+\int_{n L}^{+\infty} e^{-\sqrt{\lambda_{i}}(y-x)} u(t, y) d y\right] \\
& =\frac{\mu_{i}}{2 \sqrt{\lambda_{i}}}\left[\int_{0}^{n L} e^{-\sqrt{\lambda_{i}}(x+y)} u(t, y) d y+\int_{n L}^{+\infty} e^{-\sqrt{\lambda_{i}}(x+y)} u(t, y) d y+\int_{0}^{x} e^{-\sqrt{\lambda_{i}}(x-y)} u(t, y) d y\right] \\
& +\frac{\mu_{i}}{2 \sqrt{\lambda_{i}}}\left[\int_{x}^{n L} e^{-\sqrt{\lambda_{i}}(y-x)} u(t, y) d y+\int_{n L}^{+\infty} e^{-\sqrt{\lambda_{i}}(y-x)} u(t, y) d y\right] \\
& \leq \frac{\mu_{i}}{2 \sqrt{\lambda_{i}}}(\bar{A}+\delta)\left[\int_{0}^{n L} e^{-\sqrt{\lambda_{i}}(x+y)} d y+\int_{0}^{x} e^{-\sqrt{\lambda_{i}}(x-y)} d y+\int_{0}^{n L} e^{-\sqrt{\lambda_{i}}(y-x)} d y\right] \\
& +\frac{\mu_{i}}{2 \sqrt{\lambda_{i}}}\left[\int_{n L}^{+\infty} e^{-\sqrt{\lambda_{i}}(y-x)} u(t, y) d y+\int_{n L}^{+\infty} e^{-\sqrt{\lambda_{i}}(x+y)} u(t, y) d y\right] \\
& \leq \frac{\mu_{i}}{2 \lambda_{i}}(\bar{A}+\delta)\left[2-e^{-\sqrt{\lambda_{i}}(x+n L)}-e^{-\sqrt{\lambda_{i}}(x-n L)}\right] \\
& \left.+\frac{\mu_{i} R_{1}}{2 \sqrt{\lambda_{i}}}\left[\int_{n L}^{+\infty} e^{-\sqrt{\lambda_{i}}(y-x)} d y+\int_{n L}^{+\infty} e^{-\sqrt{\lambda_{i}}(x+y)}\right) d y\right] \\
& \left.\leq \frac{\mu_{i}}{\lambda_{i}}(\bar{A}+\delta)+\frac{\mu_{i} R_{1}}{2 \sqrt{\lambda_{i}}}\left[\int_{n L}^{+\infty} e^{-\sqrt{\lambda_{i}}(y-x)} d y+\int_{n L}^{+\infty} e^{-\sqrt{\lambda_{i}}(x+y)}\right) d y\right] \\
& =\frac{\mu_{i}}{\lambda_{i}}(\bar{A}+\delta)+\frac{\mu_{i} R_{1}}{2 \sqrt{\lambda_{i}}}\left[e^{-\sqrt{\lambda_{i}}(x+n L)}-e^{-\sqrt{\lambda_{i}}(n L-x)}\right]
\end{aligned}
$$




$$
\begin{aligned}
& =\frac{\mu_{i}}{\lambda_{i}} \bar{A}+\frac{\mu_{i}}{\lambda_{i}} \delta+\frac{\mu_{i} R_{1}}{2 \sqrt{\lambda_{i}}} e^{-\sqrt{\lambda_{i}} n L}\left(1+e^{\sqrt{\lambda_{i} L}}\right) \\
& \leq \frac{\mu_{i}}{\lambda_{i}} \bar{A}+\varepsilon
\end{aligned}
$$

for all $t>T$ and $x \in[0, L]$. The arbitrariness of $\varepsilon$ and $L$ implies that

$$
\limsup _{t \rightarrow \infty} v_{i}(t, x) \leq \frac{\mu_{i}}{\lambda_{i}} \bar{A}, \quad i=1,2 \text {, uniformly in any bounded subset of }[0,+\infty)
$$

Lemma 3.5 Let $\left(u, v_{1}, v_{2}, h\right)$ be a solution of problem (1.1). If u satisfies

$$
\liminf _{t \rightarrow \infty} u(t, x) \geq \underline{A} \quad \text { uniformly in any bounded subset of }[0,+\infty)
$$

with some constant $\underline{A}>0$, then we have

$$
\liminf _{t \rightarrow \infty} v_{i}(t, x) \geq \frac{\mu_{i}}{\lambda_{i}} \underline{A}, \quad i=1,2 \text {, uniformly in any bounded subset of }[0,+\infty)
$$

Proof For any given $L>0$ and $0<\varepsilon \ll 1$, we can choose a small positive constant $\delta$ and $k$ large enough such that

$$
\frac{\mu_{i}}{\lambda_{i}}\left[\delta+\frac{1}{2} \underline{A} e^{-\sqrt{\lambda_{i}} k L}\left(e^{-\sqrt{\lambda_{i}} L}+e^{\sqrt{\lambda_{i} L}}\right)\right]<\varepsilon .
$$

For these $k L$ and $\delta$, in view of (3.10), there exists a $T>0$ such that

$$
u(t, x) \geq \underline{A}-\delta, \quad \text { for all } t>T \text { and } x \in[0, k L] .
$$

According to (3.11), (3.12), direct calculations show that

$$
\begin{aligned}
v_{i} & (t, x) \\
& =\frac{\mu_{i}}{2 \sqrt{\lambda_{i}}}\left[\int_{-\infty}^{0} e^{-\sqrt{\lambda_{i}}|x-y|} u(t,-y) d y+\int_{0}^{+\infty} e^{-\sqrt{\lambda_{i}}|x-y|} u(t, y) d y\right] \\
& \geq \frac{\mu_{i}}{2 \sqrt{\lambda_{i}}}\left[\int_{-k L}^{0} e^{-\sqrt{\lambda_{i}}(x-y)} u(t,-y) d y+\int_{0}^{x} e^{-\sqrt{\lambda_{i}}(x-y)} u(t, y) d y+\int_{x}^{k L} e^{-\sqrt{\lambda_{i}}(y-x)} u(t, y) d y\right] \\
& \geq \frac{\mu_{i}}{2 \sqrt{\lambda_{i}}}(\underline{A}-\delta)\left[\int_{0}^{k L} e^{-\sqrt{\lambda_{i}}(x+y)} d y+\int_{0}^{x} e^{-\sqrt{\lambda_{i}}(x-y)} d y+\int_{x}^{k L} e^{-\sqrt{\lambda_{i}}(y-x)} d y\right] \\
& \geq \frac{\mu_{i}}{\lambda_{i}}(\underline{A}-\delta)\left[1-\frac{1}{2} e^{-\sqrt{\lambda_{i}} k L}\left(e^{-\sqrt{\lambda_{i}} L}+e^{\sqrt{\lambda_{i}} L}\right)\right] \\
& \geq \frac{\mu_{i}}{\lambda_{i}} \underline{A}-\frac{\mu_{i}}{\lambda_{i}}\left[\delta+\frac{1}{2} \underline{A} e^{-\sqrt{\lambda_{i}} k L}\left(e^{-\sqrt{\lambda_{i}} L}+e^{\left.\sqrt{\lambda_{i} L}\right)}\right]\right. \\
& \geq \frac{\mu_{i}}{\lambda_{i}} \underline{A}-\varepsilon
\end{aligned}
$$

for all $t>T$ and $x \in[0, L]$. The arbitrariness of $\varepsilon$ and $L$ implies that

$$
\liminf _{t \rightarrow \infty} v_{i}(t, x) \geq \frac{\mu_{i}}{\lambda_{i}} \underline{A}, \quad i=1,2 \text {, uniformly in any bounded subset of }[0,+\infty) .
$$


Theorem 3.6 Let $\left(u, v_{1}, v_{2}, h\right)$ is a solution of problem (1.1). If $h_{\infty}=\infty$ and $b>\chi_{1} \mu_{1}-$ $\chi_{2} \mu_{2}+K$, where $K$ is defined in (1.4), then we have

$$
\lim _{t \rightarrow \infty} u(t, x)=\frac{a}{b}, \quad \lim _{t \rightarrow \infty} v_{i}(t, x)=\frac{\mu_{i}}{\lambda_{i}} \frac{a}{b}, \quad i=1,2
$$

uniformly in any bounded subset of $[0,+\infty)$.

Proof We will divide our proof into two cases.

Case I. Assume that

$$
b+\chi_{2} \mu_{2}-\chi_{1} \mu_{1}-\frac{1}{\lambda_{2}}\left[\left|\chi_{2} \lambda_{2} \mu_{2}-\chi_{1} \lambda_{1} \mu_{1}\right|+\chi_{1} \mu_{1}\left|\lambda_{1}-\lambda_{2}\right|\right]>0 .
$$

Step 1 . We construct sequences by the iteration method. It follows from Lemma 2.1 that

$$
\underline{u}_{1} \triangleq 0<u(t, x) \leq \frac{a}{b+\chi_{2} \mu_{2}-\chi_{1} \mu_{1}-H_{2}} \triangleq \bar{u}_{1} \quad \text { for } t>0, x \in[0, h(t))
$$

where $\mathrm{H}_{2}$ is defined in (2.6).

(i) If $\lambda_{1} \leq \lambda_{2}$ and $\chi_{2} \mu_{2} \lambda_{2} \geq \chi_{1} \mu_{1} \lambda_{1}$, then $\frac{a}{b+\chi_{2} \mu_{2}-\chi_{1} \mu_{1}-H_{2}}=\frac{a}{b-\chi_{1} \mu_{1}\left(1-\frac{\lambda_{1}}{\lambda_{2}}\right)} \geq \frac{a}{b}$.

(ii) If $\lambda_{1} \leq \lambda_{2}$ and $\chi_{2} \mu_{2} \lambda_{2} \leq \chi_{1} \mu_{1} \lambda_{1}$, then $\frac{a}{b+\chi_{2} \mu_{2}-\chi_{1} \mu_{1}-H_{2}}=\frac{a}{b+\chi_{2} \mu_{2}-\chi_{1} \mu_{1}} \geq \frac{a}{b}$.

(iii) If $\lambda_{1} \geq \lambda_{2}$ and $\chi_{2} \mu_{2} \lambda_{2} \geq \chi_{1} \mu_{1} \lambda_{1}$, then $\frac{a}{b+\chi_{2} \mu_{2}-\chi_{1} \mu_{1}-H_{2}}=\frac{a}{b}$.

(iv) If $\lambda_{1} \geq \lambda_{2}$ and $\chi_{2} \mu_{2} \lambda_{2} \leq \chi_{1} \mu_{1} \lambda_{1}$, then $\frac{a}{b+\chi_{2} \mu_{2}-\chi_{1} \mu_{1}-H_{2}}=\frac{a}{b+\chi_{2} \mu_{2}-\chi_{1} \mu_{1} \frac{\lambda_{1}}{\lambda_{2}}} \geq \frac{a}{b}$.

By (i)-(iv), we have

$$
\bar{u}_{1} \geq \frac{a}{b}
$$

Obviously, $\bar{u}_{1}$ and $\underline{u}_{1}$ is a pair of upper and lower solution of (2.3).

Combining this with Lemmas 3.4 and 3.5, we get that

$$
\begin{array}{ll}
\limsup _{t \rightarrow \infty} v_{i}(t, x) \leq \frac{\mu_{i}}{\lambda_{i}} \bar{u}_{1} \triangleq \bar{v}_{i, 1} \quad \text { uniformly on any bounded subset of }[0,+\infty), \\
\liminf _{t \rightarrow \infty} v_{i}(t, x) \geq \frac{\mu_{i}}{\lambda_{i}} \underline{u}_{1} \triangleq \underline{v}_{i, 1} \quad \text { uniformly on any bounded subset of }[0,+\infty) .
\end{array}
$$

For every $t>0$ and $x \in[0, h(t)]$,

$$
\begin{aligned}
& \chi_{2} \lambda_{2} \nu_{2}-\chi_{1} \lambda_{1} v_{1} \\
& =\left(\chi_{2} \lambda_{2} \mu_{2}-\chi_{1} \lambda_{1} \mu_{1}\right) \int_{-h(t)}^{0} \frac{1}{2 \sqrt{\lambda_{2}}} e^{-\sqrt{\lambda_{2}}|x-y|} u(t,-y) d y \\
& \quad+\chi_{1} \lambda_{1} \mu_{1} \int_{-h(t)}^{0}\left(\frac{1}{2 \sqrt{\lambda_{2}}} e^{-\sqrt{\lambda_{2}}|x-y|}-\frac{1}{2 \sqrt{\lambda_{1}}} e^{-\sqrt{\lambda_{1}}|x-y|}\right) u(t,-y) d y \\
& \quad+\left(\chi_{2} \lambda_{2} \mu_{2}-\chi_{1} \lambda_{1} \mu_{1}\right) \int_{0}^{h(t)} \frac{1}{2 \sqrt{\lambda_{2}}} e^{-\sqrt{\lambda_{2}}|x-y|} u(t, y) d y \\
& \quad+\chi_{1} \lambda_{1} \mu_{1} \int_{0}^{h(t)}\left(\frac{1}{2 \sqrt{\lambda_{2}}} e^{-\sqrt{\lambda_{2}}|x-y|}-\frac{1}{2 \sqrt{\lambda_{1}}} e^{-\sqrt{\lambda_{1}}|x-y|}\right) u(t, y) d y
\end{aligned}
$$




$$
\begin{aligned}
\leq & \frac{1}{\lambda_{2}}\left[\left(\chi_{2} \lambda_{2} \mu_{2}-\chi_{1} \lambda_{1} \mu_{1}\right)_{+}+\chi_{1} \mu_{1}\left(\lambda_{1}-\lambda_{2}\right)_{+}\right]\left(\bar{u}_{1}+\delta\right) \\
& -\frac{1}{\lambda_{2}}\left[\left(\chi_{2} \lambda_{2} \mu_{2}-\chi_{1} \lambda_{1} \mu_{1}\right)_{-}+\chi_{1} \mu_{1}\left(\lambda_{1}-\lambda_{2}\right)_{-}\right]\left(\underline{u}_{1}-\delta\right)
\end{aligned}
$$

and

$$
\begin{aligned}
& \chi_{2} \lambda_{2} \nu_{2}-\chi_{1} \lambda_{1} \nu_{1} \\
&=\left(\chi_{2} \lambda_{2} \mu_{2}-\chi_{1} \lambda_{1} \mu_{1}\right) \int_{-h(t)}^{0} \frac{1}{2 \sqrt{\lambda_{2}}} e^{-\sqrt{\lambda_{2}}|x-y|} u(t,-y) d y \\
& \quad+\chi_{1} \lambda_{1} \mu_{1} \int_{-h(t)}^{0}\left(\frac{1}{2 \sqrt{\lambda_{2}}} e^{-\sqrt{\lambda_{2}}|x-y|}-\frac{1}{2 \sqrt{\lambda_{1}}} e^{-\sqrt{\lambda_{1}}|x-y|}\right) u(t,-y) d y \\
& \quad+\left(\chi_{2} \lambda_{2} \mu_{2}-\chi_{1} \lambda_{1} \mu_{1}\right) \int_{0}^{h(t)} \frac{1}{2 \sqrt{\lambda_{2}}} e^{-\sqrt{\lambda_{2}}|x-y|} u(t, y) d y \\
& \quad+\chi_{1} \lambda_{1} \mu_{1} \int_{0}^{h(t)}\left(\frac{1}{2 \sqrt{\lambda_{2}}} e^{-\sqrt{\lambda_{2}}|x-y|}-\frac{1}{2 \sqrt{\lambda_{1}}} e^{-\sqrt{\lambda_{1}}|x-y|}\right) u(t, y) d y \\
& \geq \frac{1}{\lambda_{2}}\left[\left(\chi_{2} \lambda_{2} \mu_{2}-\chi_{1} \lambda_{1} \mu_{1}\right)_{+}+\chi_{1} \mu_{1}\left(\lambda_{1}-\lambda_{2}\right)_{+}\right]\left(\underline{u}_{1}-\delta\right) \\
&-\frac{1}{\lambda_{2}}\left[\left(\chi_{2} \lambda_{2} \mu_{2}-\chi_{1} \lambda_{1} \mu_{1}\right)_{-}+\chi_{1} \mu_{1}\left(\lambda_{1}-\lambda_{2}\right)_{-}\right]\left(\bar{u}_{1}+\delta\right) .
\end{aligned}
$$

For any given $L>0,0<\delta \ll 1$ and $0<\varepsilon \ll 1$, let $l_{\varepsilon}$ be given by Proposition B.1 in [41]. By (3.17) and since $h_{\infty}=\infty$, there exists a $T_{1}>1$ such that $h(t)>l_{\varepsilon}$,

$$
\begin{aligned}
& \chi_{2} \lambda_{2} \nu_{2}-\chi_{1} \lambda_{1} v_{1} \\
& \leq \frac{1}{\lambda_{2}}\left[\left(\chi_{2} \lambda_{2} \mu_{2}-\chi_{1} \lambda_{1} \mu_{1}\right)_{+}+\chi_{1} \mu_{1}\left(\lambda_{1}-\lambda_{2}\right)_{+}\right]\left(\bar{u}_{1}+\delta\right) \\
& \quad-\frac{1}{\lambda_{2}}\left[\left(\chi_{2} \lambda_{2} \mu_{2}-\chi_{1} \lambda_{1} \mu_{1}\right)_{-}+\chi_{1} \mu_{1}\left(\lambda_{1}-\lambda_{2}\right)_{-}\right]\left(\underline{u}_{1}-\delta\right), \quad \forall t \geq T_{1}, x \in\left[0, l_{\varepsilon}\right] .
\end{aligned}
$$

Hence, $u$ satisfies

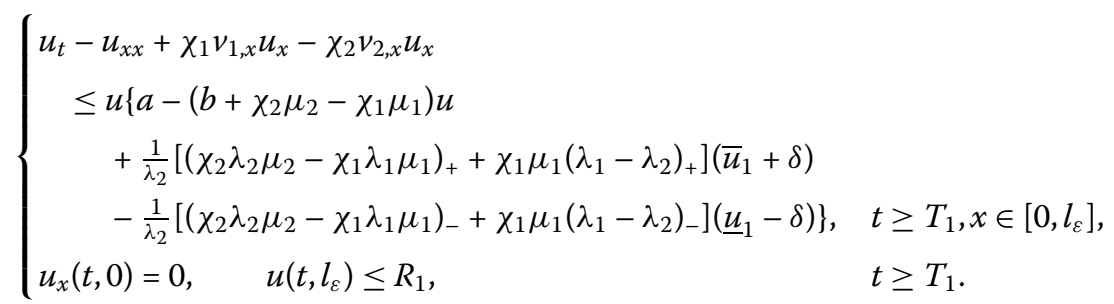

As $\bar{u}_{1} \geq \frac{a}{b}$ and $u\left(T_{1}, x\right)>0$ in $\left[0, l_{\varepsilon}\right]$, in view of Proposition B.2 in [41], this yields

$$
\begin{aligned}
& \limsup _{t \rightarrow \infty} u(t, x) \\
& \leq \frac{1}{b+\chi_{2} \mu_{2}-\chi_{1} \mu_{1}}\left\{a+\frac{1}{\lambda_{2}}\left[\left(\chi_{2} \lambda_{2} \mu_{2}-\chi_{1} \lambda_{1} \mu_{1}\right)_{+}+\chi_{1} \mu_{1}\left(\lambda_{1}-\lambda_{2}\right)_{+}\right]\left(\bar{u}_{1}+\delta\right)\right. \\
& \left.\quad-\frac{1}{\lambda_{2}}\left[\left(\chi_{2} \lambda_{2} \mu_{2}-\chi_{1} \lambda_{1} \mu_{1}\right)_{-}+\chi_{1} \mu_{1}\left(\lambda_{1}-\lambda_{2}\right)_{-}\right]\left(\underline{u}_{1}-\delta\right)\right\}+\varepsilon
\end{aligned}
$$




$$
\begin{aligned}
= & \frac{1}{b+\chi_{2} \mu_{2}-\chi_{1} \mu_{1}}\left\{a+\frac{1}{\lambda_{2}}\left[\left(\chi_{2} \lambda_{2} \mu_{2}-\chi_{1} \lambda_{1} \mu_{1}\right)_{+}+\chi_{1} \mu_{1}\left(\lambda_{1}-\lambda_{2}\right)_{+}\right]\left(\bar{u}_{1}+\delta\right)\right. \\
& \left.+\frac{1}{\lambda_{2}}\left[\left(\chi_{2} \lambda_{2} \mu_{2}-\chi_{1} \lambda_{1} \mu_{1}\right)_{-}+\chi_{1} \mu_{1}\left(\lambda_{1}-\lambda_{2}\right)_{-}\right] \delta\right\}+\varepsilon
\end{aligned}
$$

uniformly on $[0, L]$.

The arbitrariness of $\delta, \varepsilon$ and $L$ imply that

$$
\begin{aligned}
& \limsup _{t \rightarrow \infty} u(t, x) \\
& \quad \leq \frac{1}{b+\chi_{2} \mu_{2}-\chi_{1} \mu_{1}}\left\{a+\frac{1}{\lambda_{2}}\left[\left(\chi_{2} \lambda_{2} \mu_{2}-\chi_{1} \lambda_{1} \mu_{1}\right)_{+}+\chi_{1} \mu_{1}\left(\lambda_{1}-\lambda_{2}\right)_{+}\right] \bar{u}_{1}\right\} \\
& \quad \triangleq \bar{u}_{2}
\end{aligned}
$$

uniformly on any bounded subset of $[0,+\infty)$.

Combining this with Lemma 3.4, we have that

$$
\limsup _{t \rightarrow \infty} v_{i}(t, x) \leq \frac{\mu_{i}}{\lambda_{i}} \bar{u}_{2} \triangleq \bar{v}_{i, 2} \quad \text { uniformly on any bounded subset of }[0,+\infty)
$$

By (3.15) and (3.19), we obtain

$$
\begin{aligned}
\bar{u}_{1}-\bar{u}_{2}= & \bar{u}_{1}-\frac{1}{b+\chi_{2} \mu_{2}-\chi_{1} \mu_{1}}\left\{a+\frac{1}{\lambda_{2}}\left[\left(\chi_{2} \lambda_{2} \mu_{2}-\chi_{1} \lambda_{1} \mu_{1}\right)_{+}+\chi_{1} \mu_{1}\left(\lambda_{1}-\lambda_{2}\right)_{+}\right] \bar{u}_{1}\right\} \\
= & \frac{1}{b+\chi_{2} \mu_{2}-\chi_{1} \mu_{1}}\left\{\overline { u } _ { 1 } \left(b+\chi_{2} \mu_{2}-\chi_{1} \mu_{1}-\frac{1}{\lambda_{2}}\left[\left(\chi_{2} \lambda_{2} \mu_{2}-\chi_{1} \lambda_{1} \mu_{1}\right)_{+}\right.\right.\right. \\
& \left.\left.\left.+\chi_{1} \mu_{1}\left(\lambda_{1}-\lambda_{2}\right)_{+}\right]\right)-a\right\} \\
= & \frac{\bar{u}_{1}}{b+\chi_{2} \mu_{2}-\chi_{1} \mu_{1}}\left\{H_{2}-\frac{1}{\lambda_{2}}\left[\left(\chi_{2} \lambda_{2} \mu_{2}-\chi_{1} \lambda_{1} \mu_{1}\right)_{+}+\chi_{1} \mu_{1}\left(\lambda_{1}-\lambda_{2}\right)_{+}\right]\right\} \\
\geq & 0 .
\end{aligned}
$$

For any given $L>0,0<\delta \ll 1$ and $0<\varepsilon \ll 1$, let $l_{\varepsilon}$ be given by Proposition B.1 in [41]. In view of (3.18) and since $h_{\infty}=\infty$, there exists a $T_{2}>T_{1}$ such that $h(t)>l_{\varepsilon}$,

$$
\begin{aligned}
& \chi_{2} \lambda_{2} \nu_{2}-\chi_{1} \lambda_{1} v_{1} \\
& \geq \frac{1}{\lambda_{2}}\left[\left(\chi_{2} \lambda_{2} \mu_{2}-\chi_{1} \lambda_{1} \mu_{1}\right)_{+}+\chi_{1} \mu_{1}\left(\lambda_{1}-\lambda_{2}\right)_{+}\right]\left(\underline{u}_{1}-\delta\right) \\
& \quad-\frac{1}{\lambda_{2}}\left[\left(\chi_{2} \lambda_{2} \mu_{2}-\chi_{1} \lambda_{1} \mu_{1}\right)_{-}+\chi_{1} \mu_{1}\left(\lambda_{1}-\lambda_{2}\right)_{-}\right]\left(\bar{u}_{1}+\delta\right), \quad \forall t \geq T_{2}, x \in\left[0, l_{\varepsilon}\right] .
\end{aligned}
$$

Thus, $u$ satisfies

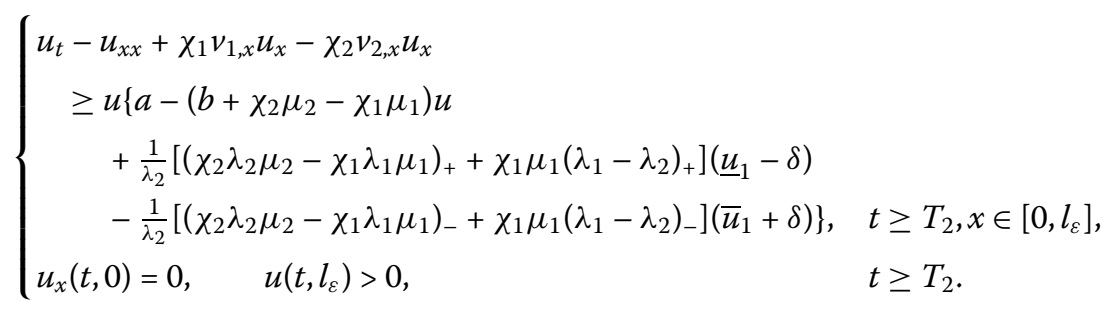


As $\bar{u}_{1} \geq \frac{a}{b}$ and $u\left(T_{2}, x\right)>0$ in $\left[0, l_{\varepsilon}\right]$, in view of Proposition B.1 in [41], this yields

$$
\begin{aligned}
\liminf _{t \rightarrow \infty} u(t, x) & \\
\geq & \frac{1}{b+\chi_{2} \mu_{2}-\chi_{1} \mu_{1}}\left\{a+\frac{1}{\lambda_{2}}\left[\left(\chi_{2} \lambda_{2} \mu_{2}-\chi_{1} \lambda_{1} \mu_{1}\right)_{+}+\chi_{1} \mu_{1}\left(\lambda_{1}-\lambda_{2}\right)_{+}\right]\left(\underline{u}_{1}-\delta\right)\right. \\
& \left.-\frac{1}{\lambda_{2}}\left[\left(\chi_{2} \lambda_{2} \mu_{2}-\chi_{1} \lambda_{1} \mu_{1}\right)_{-}+\chi_{1} \mu_{1}\left(\lambda_{1}-\lambda_{2}\right)_{-}\right]\left(\bar{u}_{1}+\delta\right)\right\}-\varepsilon \\
= & \frac{1}{b+\chi_{2} \mu_{2}-\chi_{1} \mu_{1}}\left\{a-\frac{1}{\lambda_{2}}\left[\left(\chi_{2} \lambda_{2} \mu_{2}-\chi_{1} \lambda_{1} \mu_{1}\right)_{+}+\chi_{1} \mu_{1}\left(\lambda_{1}-\lambda_{2}\right)_{+}\right] \delta\right. \\
& \left.-\frac{1}{\lambda_{2}}\left[\left(\chi_{2} \lambda_{2} \mu_{2}-\chi_{1} \lambda_{1} \mu_{1}\right)_{-}+\chi_{1} \mu_{1}\left(\lambda_{1}-\lambda_{2}\right)_{-}\right]\left(\bar{u}_{1}+\delta\right)\right\}-\varepsilon
\end{aligned}
$$

uniformly on $[0, L]$.

The arbitrariness of $\delta, \varepsilon$ and $L$ imply that

$$
\begin{aligned}
& \liminf _{t \rightarrow \infty} u(t, x) \\
& \quad \geq \frac{1}{b+\chi_{2} \mu_{2}-\chi_{1} \mu_{1}}\left\{a-\frac{1}{\lambda_{2}}\left[\left(\chi_{2} \lambda_{2} \mu_{2}-\chi_{1} \lambda_{1} \mu_{1}\right)_{-}+\chi_{1} \mu_{1}\left(\lambda_{1}-\lambda_{2}\right)_{-}\right] \bar{u}_{1}\right\} \\
& \triangleq \underline{u}_{2}
\end{aligned}
$$

uniformly on any bounded subset of $[0,+\infty)$.

Combining this with Lemma 3.5, we obtain

$$
\liminf _{t \rightarrow \infty} v_{i}(t, x) \geq \frac{\mu_{i}}{\lambda_{i}} \underline{u}_{2} \triangleq \underline{v}_{i, 2} \quad \text { uniformly on any bounded subset of }[0,+\infty)
$$

Since $\bar{u}_{1} \geq \frac{a}{b}$, we have

$$
\begin{aligned}
\underline{u}_{2}= & \frac{1}{b+\chi_{2} \mu_{2}-\chi_{1} \mu_{1}}\left\{a-\frac{1}{\lambda_{2}}\left[\left(\chi_{2} \lambda_{2} \mu_{2}-\chi_{1} \lambda_{1} \mu_{1}\right)_{-}+\chi_{1} \mu_{1}\left(\lambda_{1}-\lambda_{2}\right)_{-}\right] \bar{u}_{1}\right\} \\
\leq & \frac{1}{b+\chi_{2} \mu_{2}-\chi_{1} \mu_{1}}\left\{a+\frac{1}{\lambda_{2}}\left[\left(\chi_{2} \lambda_{2} \mu_{2}-\chi_{1} \lambda_{1} \mu_{1}\right)_{+}+\chi_{1} \mu_{1}\left(\lambda_{1}-\lambda_{2}\right)_{+}\right] \frac{a}{b}\right. \\
& \left.-\frac{1}{\lambda_{2}}\left[\left(\chi_{2} \lambda_{2} \mu_{2}-\chi_{1} \lambda_{1} \mu_{1}\right)_{-}+\chi_{1} \mu_{1}\left(\lambda_{1}-\lambda_{2}\right)_{-}\right] \bar{u}_{1}\right\} \\
\leq & \frac{1}{b+\chi_{2} \mu_{2}-\chi_{1} \mu_{1}}\left\{a+\frac{1}{\lambda_{2}}\left[\left(\chi_{2} \lambda_{2} \mu_{2}-\chi_{1} \lambda_{1} \mu_{1}\right)_{+}+\chi_{1} \mu_{1}\left(\lambda_{1}-\lambda_{2}\right)_{+}\right] \frac{a}{b}\right. \\
& \left.-\frac{1}{\lambda_{2}}\left[\left(\chi_{2} \lambda_{2} \mu_{2}-\chi_{1} \lambda_{1} \mu_{1}\right)_{-}+\chi_{1} \mu_{1}\left(\lambda_{1}-\lambda_{2}\right)_{-}\right] \frac{a}{b}\right\} \\
= & \frac{a+\frac{a}{b}\left(\chi_{2} \mu_{2}-\chi_{1} \mu_{1}\right)}{b+\chi_{2} \mu_{2}-\chi_{1} \mu_{1}}=\frac{a}{b} .
\end{aligned}
$$

Using inequality (3.14), we obtain

$$
\begin{aligned}
\underline{u}_{2} & =\frac{1}{b+\chi_{2} \mu_{2}-\chi_{1} \mu_{1}}\left\{a-\frac{1}{\lambda_{2}}\left[\left(\chi_{2} \lambda_{2} \mu_{2}-\chi_{1} \lambda_{1} \mu_{1}\right)_{-}+\chi_{1} \mu_{1}\left(\lambda_{1}-\lambda_{2}\right)_{-}\right] \bar{u}_{1}\right\} \\
& =\frac{1}{\left(b+\chi_{2} \mu_{2}-\chi_{1} \mu_{1}\right)\left(b+\chi_{2} \mu_{2}-\chi_{1} \mu_{1}-H_{2}\right)}
\end{aligned}
$$




$$
\begin{aligned}
& \times\left\{a\left(b+\chi_{2} \mu_{2}-\chi_{1} \mu_{1}-H_{2}\right)-a \frac{1}{\lambda_{2}}\left[\left(\chi_{2} \lambda_{2} \mu_{2}-\chi_{1} \lambda_{1} \mu_{1}\right)_{-}+\chi_{1} \mu_{1}\left(\lambda_{1}-\lambda_{2}\right)_{-}\right]\right\} \\
= & \frac{a}{\left(b+\chi_{2} \mu_{2}-\chi_{1} \mu_{1}\right)\left(b+\chi_{2} \mu_{2}-\chi_{1} \mu_{1}-H_{2}\right)} \\
& \times\left\{b+\chi_{2} \mu_{2}-\chi_{1} \mu_{1}-H_{2}-\frac{1}{\lambda_{2}}\left[\left(\chi_{2} \lambda_{2} \mu_{2}-\chi_{1} \lambda_{1} \mu_{1}\right)_{-}+\chi_{1} \mu_{1}\left(\lambda_{1}-\lambda_{2}\right)_{-}\right]\right\} \\
= & \frac{a}{\left(b+\chi_{2} \mu_{2}-\chi_{1} \mu_{1}\right)\left(b+\chi_{2} \mu_{2}-\chi_{1} \mu_{1}-H_{2}\right)} \\
& \times\left\{b+\chi_{2} \mu_{2}-\chi_{1} \mu_{1}\left(\frac { 1 } { \lambda _ { 2 } } \left[\left(\chi_{2} \lambda_{2} \mu_{2}-\chi_{1} \lambda_{1} \mu_{1}\right)_{+}+\chi_{1} \mu_{1}\left(\lambda_{1}-\lambda_{2}\right)_{+}\right.\right.\right. \\
& \left.\left.+\frac{1}{\lambda_{2}}\left[\left(\chi_{2} \lambda_{2} \mu_{2}-\chi_{1} \lambda_{1} \mu_{1}\right)_{-}+\chi_{1} \mu_{1}\left(\lambda_{1}-\lambda_{2}\right)_{-}\right]\right)\right\} \\
= & \frac{a}{\left(b+\chi_{2} \mu_{2}-\chi_{1} \mu_{1}\right)\left(b+\chi_{2} \mu_{2}-\chi_{1} \mu_{1}-H_{2}\right)} \\
& \times\left\{b+\chi_{2} \mu_{2}-\chi_{1} \mu_{1}-\frac{1}{\lambda_{2}}\left[\left|\chi_{2} \lambda_{2} \mu_{2}-\chi_{1} \lambda_{1} \mu_{1}\right|+\chi_{1} \mu_{1}\left|\lambda_{1}-\lambda_{2}\right|\right]\right\} \\
> & 0=\underline{u}_{1} .
\end{aligned}
$$

For any given $L>0,0<\delta \ll 1$ and $0<\varepsilon \ll 1$, let $l_{\varepsilon}$ be given by Proposition B.2 in [41]. By (3.17) and since $h_{\infty}=\infty$, there exists a $T_{3}>T_{2}$ such that $h(t)>l_{\varepsilon}$,

$$
\begin{aligned}
& \chi_{2} \lambda_{2} \nu_{2}-\chi_{1} \lambda_{1} \nu_{1} \\
& \leq \frac{1}{\lambda_{2}}\left[\left(\chi_{2} \lambda_{2} \mu_{2}-\chi_{1} \lambda_{1} \mu_{1}\right)_{+}+\chi_{1} \mu_{1}\left(\lambda_{1}-\lambda_{2}\right)_{+}\right]\left(\bar{u}_{2}+\delta\right) \\
&-\frac{1}{\lambda_{2}}\left[\left(\chi_{2} \lambda_{2} \mu_{2}-\chi_{1} \lambda_{1} \mu_{1}\right)_{-}+\chi_{1} \mu_{1}\left(\lambda_{1}-\lambda_{2}\right)_{-}\right]\left(\underline{u}_{2}-\delta\right), \quad \forall t \geq T_{3}, x \in\left[0, l_{\varepsilon}\right] .
\end{aligned}
$$

Hence, $u$ satisfies

$$
\left\{\begin{array}{rlr}
u_{t}- & u_{x x}+\chi_{1} v_{1, x} u_{x}-\chi_{2} \nu_{2, x} u_{x} & \\
\leq & u\left\{a-\left(b+\chi_{2} \mu_{2}-\chi_{1} \mu_{1}\right) u\right. & \\
& \quad+\frac{1}{\lambda_{2}}\left[\left(\chi_{2} \lambda_{2} \mu_{2}-\chi_{1} \lambda_{1} \mu_{1}\right)_{+}+\chi_{1} \mu_{1}\left(\lambda_{1}-\lambda_{2}\right)_{+}\right]\left(\bar{u}_{2}+\delta\right) & \\
\left.\quad-\frac{1}{\lambda_{2}}\left[\left(\chi_{2} \lambda_{2} \mu_{2}-\chi_{1} \lambda_{1} \mu_{1}\right)_{-}+\chi_{1} \mu_{1}\left(\lambda_{1}-\lambda_{2}\right)_{-}\right]\left(\underline{u}_{2}-\delta\right)\right\}, & t \geq T_{3}, x \in\left[0, l_{\varepsilon}\right], \\
u_{x}(t, 0)=0, \quad u\left(t, l_{\varepsilon}\right) \leq R_{1}, & t \geq T_{3} .
\end{array}\right.
$$

As $\bar{u}_{2} \geq \frac{a}{b}, \underline{u}_{1} \leq \frac{a}{b}$ and $u\left(T_{3}, x\right)>0$ in $\left[0, l_{\varepsilon}\right]$, in view of Proposition B.2 in [41], this yields

$$
\begin{aligned}
\limsup _{t \rightarrow \infty} u(t, x) & \\
\leq & \frac{1}{b+\chi_{2} \mu_{2}-\chi_{1} \mu_{1}}\left\{a+\frac{1}{\lambda_{2}}\left[\left(\chi_{2} \lambda_{2} \mu_{2}-\chi_{1} \lambda_{1} \mu_{1}\right)_{+}+\chi_{1} \mu_{1}\left(\lambda_{1}-\lambda_{2}\right)_{+}\right]\left(\bar{u}_{2}+\delta\right)\right. \\
& \left.\quad-\frac{1}{\lambda_{2}}\left[\left(\chi_{2} \lambda_{2} \mu_{2}-\chi_{1} \lambda_{1} \mu_{1}\right)_{-}+\chi_{1} \mu_{1}\left(\lambda_{1}-\lambda_{2}\right)_{-}\right]\left(\underline{u}_{2}-\delta\right)\right\}+\varepsilon
\end{aligned}
$$

uniformly on $[0, L]$. 
The arbitrariness of $\delta, \varepsilon$ and $L$ imply that

$$
\begin{aligned}
\limsup _{t \rightarrow \infty} u(t, x) & \\
\leq & \frac{1}{b+\chi_{2} \mu_{2}-\chi_{1} \mu_{1}}\left\{a+\frac{1}{\lambda_{2}}\left[\left(\chi_{2} \lambda_{2} \mu_{2}-\chi_{1} \lambda_{1} \mu_{1}\right)_{+}+\chi_{1} \mu_{1}\left(\lambda_{1}-\lambda_{2}\right)_{+}\right] \bar{u}_{2}\right. \\
& \left.\quad-\frac{1}{\lambda_{2}}\left[\left(\chi_{2} \lambda_{2} \mu_{2}-\chi_{1} \lambda_{1} \mu_{1}\right)_{-}+\chi_{1} \mu_{1}\left(\lambda_{1}-\lambda_{2}\right)_{-}\right] \underline{u}_{2}\right\} \\
\triangleq & \bar{u}_{3}
\end{aligned}
$$

uniformly on any bounded subset of $[0,+\infty)$.

Combining this with Lemma 3.4, we have

$$
\underset{t \rightarrow \infty}{\limsup } v_{i}(t, x) \leq \frac{\mu_{i}}{\lambda_{i}} \bar{u}_{3} \triangleq \bar{v}_{i, 3} \quad \text { uniformly on any bounded subset of }[0,+\infty) .
$$

Since $\bar{u}_{2} \geq \frac{a}{b}$ and $\underline{u}_{2} \leq \frac{a}{b}$, we find that

$$
\begin{aligned}
\bar{u}_{3}= & \frac{1}{b+\chi_{2} \mu_{2}-\chi_{1} \mu_{1}}\left\{a+\frac{1}{\lambda_{2}}\left[\left(\chi_{2} \lambda_{2} \mu_{2}-\chi_{1} \lambda_{1} \mu_{1}\right)_{+}+\chi_{1} \mu_{1}\left(\lambda_{1}-\lambda_{2}\right)_{+}\right] \bar{u}_{2}\right. \\
& \left.-\frac{1}{\lambda_{2}}\left[\left(\chi_{2} \lambda_{2} \mu_{2}-\chi_{1} \lambda_{1} \mu_{1}\right)_{-}+\chi_{1} \mu_{1}\left(\lambda_{1}-\lambda_{2}\right)_{-}\right] \underline{u}_{2}\right\} \\
\geq & \frac{1}{b+\chi_{2} \mu_{2}-\chi_{1} \mu_{1}}\left\{a+\frac{1}{\lambda_{2}}\left[\left(\chi_{2} \lambda_{2} \mu_{2}-\chi_{1} \lambda_{1} \mu_{1}\right)_{+}+\chi_{1} \mu_{1}\left(\lambda_{1}-\lambda_{2}\right)_{+}\right] \frac{a}{b}\right. \\
& \left.-\frac{1}{\lambda_{2}}\left[\left(\chi_{2} \lambda_{2} \mu_{2}-\chi_{1} \lambda_{1} \mu_{1}\right)_{-}+\chi_{1} \mu_{1}\left(\lambda_{1}-\lambda_{2}\right)_{-}\right] \frac{a}{b}\right\} \\
= & \frac{a-\chi_{1} \mu_{1} \frac{a}{b}+\chi_{2} \mu_{2} \frac{a}{b}}{\chi_{2} \mu_{2}+b-\chi_{1} \mu_{1}} \\
= & \frac{a}{b} .
\end{aligned}
$$

Using inequality (3.20), we obtain

$$
\begin{aligned}
\bar{u}_{2}-\bar{u}_{3}= & \frac{1}{b+\chi_{2} \mu_{2}-\chi_{1} \mu_{1}}\left\{a+\frac{1}{\lambda_{2}}\left[\left(\chi_{2} \lambda_{2} \mu_{2}-\chi_{1} \lambda_{1} \mu_{1}\right)_{+}+\chi_{1} \mu_{1}\left(\lambda_{1}-\lambda_{2}\right)_{+}\right] \bar{u}_{1}\right\} \\
& -\frac{1}{b+\chi_{2} \mu_{2}-\chi_{1} \mu_{1}}\left\{a+\frac{1}{\lambda_{2}}\left[\left(\chi_{2} \lambda_{2} \mu_{2}-\chi_{1} \lambda_{1} \mu_{1}\right)_{+}+\chi_{1} \mu_{1}\left(\lambda_{1}-\lambda_{2}\right)_{+}\right] \bar{u}_{2}\right. \\
& \left.-\frac{1}{\lambda_{2}}\left[\left(\chi_{2} \lambda_{2} \mu_{2}-\chi_{1} \lambda_{1} \mu_{1}\right)_{-}+\chi_{1} \mu_{1}\left(\lambda_{1}-\lambda_{2}\right)_{-}\right] \underline{u}_{2}\right\} \\
= & \frac{1}{b+\chi_{2} \mu_{2}-\chi_{1} \mu_{1}}\left\{\frac{1}{\lambda_{2}}\left[\left(\chi_{2} \lambda_{2} \mu_{2}-\chi_{1} \lambda_{1} \mu_{1}\right)_{+}+\chi_{1} \mu_{1}\left(\lambda_{1}-\lambda_{2}\right)_{+}\right]\left(\bar{u}_{1}-\bar{u}_{2}\right)\right. \\
& \left.+\frac{1}{\lambda_{2}}\left[\left(\chi_{2} \lambda_{2} \mu_{2}-\chi_{1} \lambda_{1} \mu_{1}\right)_{-}+\chi_{1} \mu_{1}\left(\lambda_{1}-\lambda_{2}\right)_{-}\right] \underline{u}_{2}\right\} \\
\geq & 0 .
\end{aligned}
$$


For any given $L>0,0<\delta \ll 1$ and $0<\varepsilon \ll 1$, let $l_{\varepsilon}$ be given by Proposition B.1 in [41]. In view of (3.18) and since $h_{\infty}=\infty$, there exists a $T_{4}>T_{3}$ such that $h(t)>l_{\varepsilon}$,

$$
\begin{aligned}
& \chi_{2} \lambda_{2} v_{2}-\chi_{1} \lambda_{1} v_{1} \\
& \geq \frac{1}{\lambda_{2}}\left[\left(\chi_{2} \lambda_{2} \mu_{2}-\chi_{1} \lambda_{1} \mu_{1}\right)_{+}+\chi_{1} \mu_{1}\left(\lambda_{1}-\lambda_{2}\right)_{+}\right]\left(\underline{u}_{2}-\delta\right) \\
& \quad-\frac{1}{\lambda_{2}}\left[\left(\chi_{2} \lambda_{2} \mu_{2}-\chi_{1} \lambda_{1} \mu_{1}\right)_{-}+\chi_{1} \mu_{1}\left(\lambda_{1}-\lambda_{2}\right)_{-}\right]\left(\bar{u}_{2}+\delta\right), \quad \forall t \geq T_{4}, x \in\left[0, l_{\varepsilon}\right] .
\end{aligned}
$$

Thus, $u$ satisfies

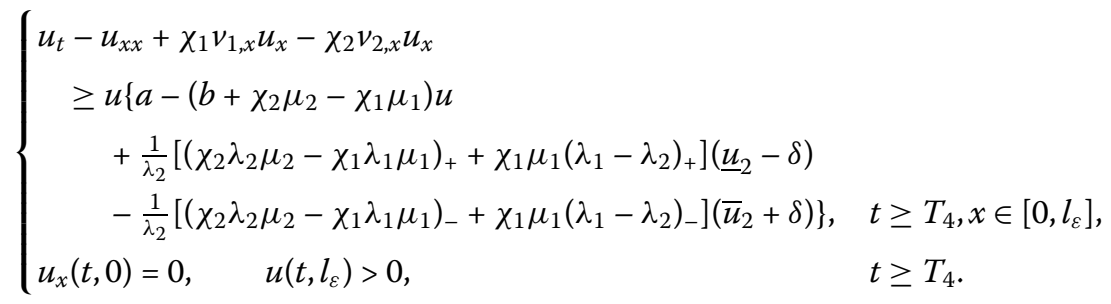

As $\bar{u}_{2} \geq \frac{a}{b}, \underline{u}_{1} \leq \frac{a}{b}$ and $u\left(T_{4}, x\right)>0$ in $\left[0, l_{\varepsilon}\right]$, in view of Proposition B.1 in [41], this yields

$$
\begin{aligned}
& \liminf _{t \rightarrow \infty} u(t, x) \\
& \geq \frac{1}{b+\chi_{2} \mu_{2}-\chi_{1} \mu_{1}}\left\{a+\frac{1}{\lambda_{2}}\left[\left(\chi_{2} \lambda_{2} \mu_{2}-\chi_{1} \lambda_{1} \mu_{1}\right)_{+}+\chi_{1} \mu_{1}\left(\lambda_{1}-\lambda_{2}\right)_{+}\right]\left(\underline{u}_{2}-\delta\right)\right. \\
& \left.\quad-\frac{1}{\lambda_{2}}\left[\left(\chi_{2} \lambda_{2} \mu_{2}-\chi_{1} \lambda_{1} \mu_{1}\right)_{-}+\chi_{1} \mu_{1}\left(\lambda_{1}-\lambda_{2}\right)_{-}\right]\left(\bar{u}_{2}+\delta\right)\right\}-\varepsilon
\end{aligned}
$$

uniformly on $[0, L]$.

The arbitrariness of $\delta, \varepsilon$ and $L$ imply that

$$
\begin{aligned}
\liminf _{t \rightarrow \infty} u(t, x) \geq & \frac{1}{b+\chi_{2} \mu_{2}-\chi_{1} \mu_{1}}\left\{a+\frac{1}{\lambda_{2}}\left[\left(\chi_{2} \lambda_{2} \mu_{2}-\chi_{1} \lambda_{1} \mu_{1}\right)_{+}+\chi_{1} \mu_{1}\left(\lambda_{1}-\lambda_{2}\right)_{+}\right] \underline{u}_{2}\right. \\
& \left.-\frac{1}{\lambda_{2}}\left[\left(\chi_{2} \lambda_{2} \mu_{2}-\chi_{1} \lambda_{1} \mu_{1}\right)_{-}+\chi_{1} \mu_{1}\left(\lambda_{1}-\lambda_{2}\right)_{-}\right] \bar{u}_{2}\right\} \triangleq \underline{u}_{3}
\end{aligned}
$$

uniformly on any bounded subset of $[0,+\infty)$.

Combining this with Lemma 3.5, we have

$$
\liminf _{t \rightarrow \infty} v_{i}(t, x) \geq \frac{\mu_{i}}{\lambda_{i}} \underline{u}_{3} \triangleq \underline{v}_{i, 3} \quad \text { uniformly on any bounded subset of }[0,+\infty)
$$

Since $\bar{u}_{2} \geq \frac{a}{b}$ and $\underline{u}_{2} \leq \frac{a}{b}$, we obtain that

$$
\begin{aligned}
\underline{u}_{3}= & \frac{1}{b+\chi_{2} \mu_{2}-\chi_{1} \mu_{1}}\left\{a+\frac{1}{\lambda_{2}}\left[\left(\chi_{2} \lambda_{2} \mu_{2}-\chi_{1} \lambda_{1} \mu_{1}\right)_{+}+\chi_{1} \mu_{1}\left(\lambda_{1}-\lambda_{2}\right)_{+}\right] \underline{u}_{2}\right. \\
& \left.-\frac{1}{\lambda_{2}}\left[\left(\chi_{2} \lambda_{2} \mu_{2}-\chi_{1} \lambda_{1} \mu_{1}\right)_{-}+\chi_{1} \mu_{1}\left(\lambda_{1}-\lambda_{2}\right)_{-}\right] \bar{u}_{2}\right\} \\
\leq & \frac{1}{b+\chi_{2} \mu_{2}-\chi_{1} \mu_{1}}\left\{a+\frac{1}{\lambda_{2}}\left[\left(\chi_{2} \lambda_{2} \mu_{2}-\chi_{1} \lambda_{1} \mu_{1}\right)_{+}+\chi_{1} \mu_{1}\left(\lambda_{1}-\lambda_{2}\right)_{+}\right] \frac{a}{b}\right.
\end{aligned}
$$




$$
\begin{aligned}
& \left.-\frac{1}{\lambda_{2}}\left[\left(\chi_{2} \lambda_{2} \mu_{2}-\chi_{1} \lambda_{1} \mu_{1}\right)_{-}+\chi_{1} \mu_{1}\left(\lambda_{1}-\lambda_{2}\right)_{-}\right] \frac{a}{b}\right\} \\
= & \frac{a-\chi_{1} \mu_{1} \frac{a}{b}+\chi_{2} \mu_{2} \frac{a}{b}}{\chi_{2} \mu_{2}+b-\chi_{1} \mu_{1}}=\frac{a}{b} .
\end{aligned}
$$

Using inequality (3.20), we have

$$
\begin{aligned}
\underline{u}_{2}-\underline{u}_{3}= & \frac{1}{b+\chi_{2} \mu_{2}-\chi_{1} \mu_{1}}\left\{a-\frac{1}{\lambda_{2}}\left[\left(\chi_{2} \lambda_{2} \mu_{2}-\chi_{1} \lambda_{1} \mu_{1}\right)_{-}+\chi_{1} \mu_{1}\left(\lambda_{1}-\lambda_{2}\right)_{-}\right] \bar{u}_{1}\right\} \\
& -\frac{1}{b+\chi_{2} \mu_{2}-\chi_{1} \mu_{1}}\left\{a+\frac{1}{\lambda_{2}}\left[\left(\chi_{2} \lambda_{2} \mu_{2}-\chi_{1} \lambda_{1} \mu_{1}\right)_{+}+\chi_{1} \mu_{1}\left(\lambda_{1}-\lambda_{2}\right)_{+}\right] \underline{u}_{2}\right. \\
& \left.-\frac{1}{\lambda_{2}}\left[\left(\chi_{2} \lambda_{2} \mu_{2}-\chi_{1} \lambda_{1} \mu_{1}\right)_{-}+\chi_{1} \mu_{1}\left(\lambda_{1}-\lambda_{2}\right)_{-}\right] \bar{u}_{2}\right\} \\
= & \frac{1}{b+\chi_{2} \mu_{2}-\chi_{1} \mu_{1}}\left\{-\frac{1}{\lambda_{2}}\left[\left(\chi_{2} \lambda_{2} \mu_{2}-\chi_{1} \lambda_{1} \mu_{1}\right)_{-}+\chi_{1} \mu_{1}\left(\lambda_{1}-\lambda_{2}\right)_{-}\right]\left(\bar{u}_{1}-\bar{u}_{2}\right)\right. \\
& \left.-\frac{1}{\lambda_{2}}\left[\left(\chi_{2} \lambda_{2} \mu_{2}-\chi_{1} \lambda_{1} \mu_{1}\right)_{+}+\chi_{1} \mu_{1}\left(\lambda_{1}-\lambda_{2}\right)_{+}\right] \underline{u}_{2}\right\} \\
\leq & 0 .
\end{aligned}
$$

Repeating the above procedure, we can find six sequences $\left\{\underline{u}_{n}\right\},\left\{\bar{u}_{n}\right\},\left\{\underline{v}_{i, n}\right\},\left\{\bar{v}_{i, n}\right\}$ such that for all $n$,

$$
\begin{aligned}
& \underline{u}_{n} \leq \liminf _{t \rightarrow \infty} u(t, x) \leq \limsup _{t \rightarrow \infty} u(t, x) \leq \bar{u}_{n}, \\
& \underline{v}_{i, n} \triangleq \frac{\mu_{i}}{\lambda_{i}} \underline{u}_{n} \leq \liminf _{t \rightarrow \infty} v_{i}(t, x) \leq \limsup _{t \rightarrow \infty} v_{i}(t, x) \leq \frac{\mu_{i}}{\lambda_{i}} \bar{u}_{n} \triangleq \bar{v}_{i, n}, \quad i=1,2
\end{aligned}
$$

uniformly on any bounded subset of $[0,+\infty)$.

Moreover, these sequences can be determined by the following iterative formulas:

$$
\left\{\begin{aligned}
\bar{u}_{n}= & \frac{1}{b+\chi_{2} \mu_{2}-\chi_{1} \mu_{1}}\left\{a+\frac{1}{\lambda_{2}}\left[\left(\chi_{2} \lambda_{2} \mu_{2}-\chi_{1} \lambda_{1} \mu_{1}\right)_{+}+\chi_{1} \mu_{1}\left(\lambda_{1}-\lambda_{2}\right)_{+}\right] \bar{u}_{n-1}\right. \\
& \left.-\frac{1}{\lambda_{2}}\left[\left(\chi_{2} \lambda_{2} \mu_{2}-\chi_{1} \lambda_{1} \mu_{1}\right)_{-}+\chi_{1} \mu_{1}\left(\lambda_{1}-\lambda_{2}\right)_{-}\right] \underline{u}_{n-1}\right\}, \quad n=2,3, \ldots \\
\underline{u}_{n}= & \frac{1}{b+\chi_{2} \mu_{2}-\chi_{1} \mu_{1}}\left\{a+\frac{1}{\lambda_{2}}\left[\left(\chi_{2} \lambda_{2} \mu_{2}-\chi_{1} \lambda_{1} \mu_{1}\right)_{+}+\chi_{1} \mu_{1}\left(\lambda_{1}-\lambda_{2}\right)_{+}\right] \underline{u}_{n-1}\right. \\
& \left.-\frac{1}{\lambda_{2}}\left[\left(\chi_{2} \lambda_{2} \mu_{2}-\chi_{1} \lambda_{1} \mu_{1}\right)_{-}+\chi_{1} \mu_{1}\left(\lambda_{1}-\lambda_{2}\right)_{-}\right] \bar{u}_{n-1}\right\}, \quad n=2,3, \ldots
\end{aligned}\right.
$$

Step 2. We prove the convergence of sequences $\left\{\underline{u}_{n}\right\}$ and $\left\{\bar{u}_{n}\right\}$ by the monotone convergence theorem.

(i) First, we prove that $\left\{\underline{u}_{n}\right\}$ and $\left\{\bar{u}_{n}\right\}$ are bounded sequences by induction.

The trivial case is $n=1$ since $\underline{u}_{1} \leq \frac{a}{b} \leq \bar{u}_{1}$ by (3.15) and (3.16). Assume that it is true for some $n \geq 1$, namely,

$$
\underline{u}_{j} \leq \frac{a}{b} \leq \bar{u}_{j}, \quad j=1,2, \ldots, n .
$$


Next we will prove that for $n+1, \underline{u}_{n+1} \leq \frac{a}{b} \leq \bar{u}_{n+1}$. According to (3.30) and (3.31), we have

$$
\begin{aligned}
\bar{u}_{n+1}= & \frac{1}{b+\chi_{2} \mu_{2}-\chi_{1} \mu_{1}}\left\{a+\frac{1}{\lambda_{2}}\left[\left(\chi_{2} \lambda_{2} \mu_{2}-\chi_{1} \lambda_{1} \mu_{1}\right)_{+}+\chi_{1} \mu_{1}\left(\lambda_{1}-\lambda_{2}\right)_{+}\right] \bar{u}_{n}\right. \\
& \left.-\frac{1}{\lambda_{2}}\left[\left(\chi_{2} \lambda_{2} \mu_{2}-\chi_{1} \lambda_{1} \mu_{1}\right)_{-}+\chi_{1} \mu_{1}\left(\lambda_{1}-\lambda_{2}\right)_{-}\right] \underline{u}_{n}\right\} \\
\geq & \frac{a-\chi_{1} \mu_{1} \frac{a}{b}+\chi_{2} \mu_{2} \frac{a}{b}}{b+\chi_{2} \mu_{2}-\chi_{1} \mu_{1}}=\frac{a}{b}, \\
\underline{u}_{n+1}= & \frac{1}{b+\chi_{2} \mu_{2}-\chi_{1} \mu_{1}}\left\{a+\frac{1}{\lambda_{2}}\left[\left(\chi_{2} \lambda_{2} \mu_{2}-\chi_{1} \lambda_{1} \mu_{1}\right)_{+}+\chi_{1} \mu_{1}\left(\lambda_{1}-\lambda_{2}\right)_{+}\right] \underline{u}_{n}\right. \\
& \left.-\frac{1}{\lambda_{2}}\left[\left(\chi_{2} \lambda_{2} \mu_{2}-\chi_{1} \lambda_{1} \mu_{1}\right)_{-}+\chi_{1} \mu_{1}\left(\lambda_{1}-\lambda_{2}\right)_{-}\right] \bar{u}_{n}\right\} \\
\leq & \frac{a-\chi_{1} \mu_{1} \frac{a}{b}+\chi_{2} \mu_{2} \frac{a}{b}}{b+\chi_{2} \mu_{2}-\chi_{1} \mu_{1}}=\frac{a}{b} .
\end{aligned}
$$

Thus, by induction, for all $n$, we have that $\underline{u}_{n} \leq \frac{a}{b} \leq \bar{u}_{n}$.

(ii) Second, using a similar argument as above, we can prove that sequence $\left\{\underline{u}_{n}\right\}$ is increasing and $\left\{\bar{u}_{n}\right\}$ is decreasing in $n$.

In the same way, by (3.20) and (3.23), we have

$$
\bar{u}_{1} \geq \bar{u}_{2}, \quad \underline{u}_{1} \leq \underline{u}_{2} .
$$

Suppose that it is true for some $n \geq 2$, that is,

$$
\bar{u}_{j-1} \geq \bar{u}_{j}, \quad \underline{u}_{j-1} \leq \underline{u}_{j}, \quad j=2,3, \ldots, n .
$$

We will prove that for $n+1, \bar{u}_{n} \geq \bar{u}_{n+1}$ and $\underline{u}_{n} \leq \underline{u}_{n+1}$. From (3.30) and (3.33) we finally establish

$$
\begin{aligned}
\bar{u}_{n}= & \frac{1}{b+\chi_{2} \mu_{2}-\chi_{1} \mu_{1}}\left\{a+\frac{1}{\lambda_{2}}\left[\left(\chi_{2} \lambda_{2} \mu_{2}-\chi_{1} \lambda_{1} \mu_{1}\right)_{+}+\chi_{1} \mu_{1}\left(\lambda_{1}-\lambda_{2}\right)_{+}\right] \bar{u}_{n-1}\right. \\
& \left.-\frac{1}{\lambda_{2}}\left[\left(\chi_{2} \lambda_{2} \mu_{2}-\chi_{1} \lambda_{1} \mu_{1}\right)_{-}+\chi_{1} \mu_{1}\left(\lambda_{1}-\lambda_{2}\right)_{-}\right] \underline{u}_{n-1}\right\} \\
\geq & \frac{1}{b+\chi_{2} \mu_{2}-\chi_{1} \mu_{1}}\left\{a+\frac{1}{\lambda_{2}}\left[\left(\chi_{2} \lambda_{2} \mu_{2}-\chi_{1} \lambda_{1} \mu_{1}\right)_{+}+\chi_{1} \mu_{1}\left(\lambda_{1}-\lambda_{2}\right)_{+}\right] \bar{u}_{n}\right. \\
& \left.-\frac{1}{\lambda_{2}}\left[\left(\chi_{2} \lambda_{2} \mu_{2}-\chi_{1} \lambda_{1} \mu_{1}\right)_{-}+\chi_{1} \mu_{1}\left(\lambda_{1}-\lambda_{2}\right)_{-}\right] \underline{u}_{n}\right\}=\bar{u}_{n+1}, \quad n=2,3, \ldots, \\
\underline{u}_{n}= & \frac{1}{b+\chi_{2} \mu_{2}-\chi_{1} \mu_{1}}\left\{a+\frac{1}{\lambda_{2}}\left[\left(\chi_{2} \lambda_{2} \mu_{2}-\chi_{1} \lambda_{1} \mu_{1}\right)_{+}+\chi_{1} \mu_{1}\left(\lambda_{1}-\lambda_{2}\right)_{+}\right] \underline{u}_{n-1}\right. \\
& \left.-\frac{1}{\lambda_{2}}\left[\left(\chi_{2} \lambda_{2} \mu_{2}-\chi_{1} \lambda_{1} \mu_{1}\right)_{-}+\chi_{1} \mu_{1}\left(\lambda_{1}-\lambda_{2}\right)_{-}\right] \bar{u}_{n-1}\right\} \\
\leq & \frac{1}{b+\chi_{2} \mu_{2}-\chi_{1} \mu_{1}}\left\{a+\frac{1}{\lambda_{2}}\left[\left(\chi_{2} \lambda_{2} \mu_{2}-\chi_{1} \lambda_{1} \mu_{1}\right)_{+}+\chi_{1} \mu_{1}\left(\lambda_{1}-\lambda_{2}\right)_{+}\right] \underline{u}_{n}\right. \\
& \left.-\frac{1}{\lambda_{2}}\left[\left(\chi_{2} \lambda_{2} \mu_{2}-\chi_{1} \lambda_{1} \mu_{1}\right)_{-}+\chi_{1} \mu_{1}\left(\lambda_{1}-\lambda_{2}\right)_{-}\right] \bar{u}_{n}\right\}=\underline{u}_{n+1}, \quad n=2,3, \ldots
\end{aligned}
$$

Therefore, by induction, for all $n$, we obtain that $\bar{u}_{n-1} \geq \bar{u}_{n}$ and $\underline{u}_{n-1} \leq \underline{u}_{n}$. 
Combining (i) and (ii), we derive that sequences $\left\{\underline{u}_{n}\right\}$ and $\left\{\bar{u}_{n}\right\}$ are monotonic and bounded. It is well known that sequences $\left\{\underline{u}_{n}\right\}$ and $\left\{\bar{u}_{n}\right\}$ converge. Thus there exist positive constants $h$ and $H$ such that

$$
\lim _{n \rightarrow \infty} \underline{u}_{n}=h, \quad \lim _{n \rightarrow \infty} \bar{u}_{n}=H .
$$

Step 3. We complete the proof. Passing to limits in equations (3.30), it is easy to see that $h$ and $H$ satisfy the following equation:

$$
\begin{aligned}
H= & \frac{1}{b+\chi_{2} \mu_{2}-\chi_{1} \mu_{1}}\left\{a+\frac{1}{\lambda_{2}}\left[\left(\chi_{2} \lambda_{2} \mu_{2}-\chi_{1} \lambda_{1} \mu_{1}\right)_{+}+\chi_{1} \mu_{1}\left(\lambda_{1}-\lambda_{2}\right)_{+}\right] H\right. \\
& \left.-\frac{1}{\lambda_{2}}\left[\left(\chi_{2} \lambda_{2} \mu_{2}-\chi_{1} \lambda_{1} \mu_{1}\right)_{-}+\chi_{1} \mu_{1}\left(\lambda_{1}-\lambda_{2}\right)_{-}\right] h\right\}, \\
h= & \frac{1}{b+\chi_{2} \mu_{2}-\chi_{1} \mu_{1}}\left\{a+\frac{1}{\lambda_{2}}\left[\left(\chi_{2} \lambda_{2} \mu_{2}-\chi_{1} \lambda_{1} \mu_{1}\right)_{+}+\chi_{1} \mu_{1}\left(\lambda_{1}-\lambda_{2}\right)_{+}\right] h\right. \\
& \left.-\frac{1}{\lambda_{2}}\left[\left(\chi_{2} \lambda_{2} \mu_{2}-\chi_{1} \lambda_{1} \mu_{1}\right)_{-}+\chi_{1} \mu_{1}\left(\lambda_{1}-\lambda_{2}\right)_{-}\right] H\right\} .
\end{aligned}
$$

By a straightforward calculation, we obtain that both $h$ and $H$ satisfy

$$
h=H=\frac{a}{b} \text {. }
$$

Therefore, we conclude that

$$
\lim _{t \rightarrow \infty} u(t, x)=\frac{a}{b} \quad \text { uniformly in any bounded subset of }[0,+\infty) .
$$

Combining (3.29), (3.35) and (3.37), we have

$$
\lim _{t \rightarrow \infty} v_{i}(t, x)=\frac{\mu_{i}}{\lambda_{i}} \frac{a}{b} \quad \text { uniformly in any bounded subset of }[0,+\infty) .
$$

This completes the first case.

Case II. Assume that

$$
b+\chi_{2} \mu_{2}-\chi_{1} \mu_{1}-\frac{1}{\lambda_{1}}\left[\left|\chi_{2} \lambda_{2} \mu_{2}-\chi_{1} \lambda_{1} \mu_{1}\right|+\chi_{2} \mu_{2}\left|\lambda_{1}-\lambda_{2}\right|\right]>0 .
$$

It follows from Lemma 2.1 that

$$
\underline{u}_{1} \triangleq 0<u(t, x) \leq \frac{a}{b+\chi_{2} \mu_{2}-\chi_{1} \mu_{1}-H_{1}} \triangleq \bar{u}_{1} \quad \text { for } t>0, x \in[0, h(t)),
$$

where $H_{1}$ is defined in (2.6).

(i) If $\lambda_{1} \leq \lambda_{2}$ and $\chi_{2} \mu_{2} \lambda_{2} \geq \chi_{1} \mu_{1} \lambda_{1}$, then $\frac{a}{b+\chi_{2} \mu_{2}-\chi_{1} \mu_{1}-H_{2}}=\frac{a}{b+\chi_{2} \mu_{2}\left(1-\frac{\lambda_{2}}{\lambda_{1}}\right)} \geq \frac{a}{b}$.

(ii) If $\lambda_{1} \leq \lambda_{2}$ and $\chi_{2} \mu_{2} \lambda_{2} \leq \chi_{1} \mu_{1} \lambda_{1}$, then $\frac{a}{b+\chi_{2} \mu_{2}-\chi_{1} \mu_{1}-H_{2}}=\frac{a}{b+\chi_{2} \mu_{2}-\chi_{1} \mu_{1}} \geq \frac{a}{b}$.

(iii) If $\lambda_{1} \geq \lambda_{2}$ and $\chi_{2} \mu_{2} \lambda_{2} \geq \chi_{1} \mu_{1} \lambda_{1}$, then $\frac{a}{b+\chi_{2} \mu_{2}-\chi_{1} \mu_{1}-H_{2}}=\frac{a}{b}$.

(iv) If $\lambda_{1} \geq \lambda_{2}$ and $\chi_{2} \mu_{2} \lambda_{2} \leq \chi_{1} \mu_{1} \lambda_{1}$, then $\frac{a}{b+\chi_{2} \mu_{2}-\chi_{1} \mu_{1}-H_{2}}=\frac{a}{b+\chi_{2} \mu_{2} \frac{\lambda_{2}}{\lambda_{1}}-\chi_{1} \mu_{1}} \geq \frac{a}{b}$. 
By (i)-(iv), we have

$$
\bar{u}_{1} \geq \frac{a}{b} .
$$

Rewrite $\chi_{2} \lambda_{2} \nu_{2}-\chi_{1} \lambda_{1} v_{1}$ in the form

$$
\begin{aligned}
& \chi_{2} \lambda_{2} \nu_{2}-\chi_{1} \lambda_{1} \nu_{1} \\
&=\left(\chi_{2} \lambda_{2} \mu_{2}-\chi_{1} \lambda_{1} \mu_{1}\right) \int_{-h(t)}^{0} \frac{1}{2 \sqrt{\lambda_{1}}} e^{-\sqrt{\lambda_{1}}|x-y|} w(t,-y) d y \\
& \quad+\chi_{2} \lambda_{2} \mu_{2} \int_{-h(t)}^{0}\left(\frac{1}{2 \sqrt{\lambda_{2}}} e^{-\sqrt{\lambda_{2}}|x-y|}-\frac{1}{2 \sqrt{\lambda_{1}}} e^{-\sqrt{\lambda_{1}}|x-y|}\right) w(t,-y) d y \\
& \quad+\left(\chi_{2} \lambda_{2} \mu_{2}-\chi_{1} \lambda_{1} \mu_{1}\right) \int_{0}^{h(t)} \frac{1}{2 \sqrt{\lambda_{1}}} e^{-\sqrt{\lambda_{1}}|x-y|} w(t, y) d y \\
& \quad+\chi_{2} \lambda_{2} \mu_{2} \int_{0}^{h(t)}\left(\frac{1}{2 \sqrt{\lambda_{2}}} e^{-\sqrt{\lambda_{2}}|x-y|}-\frac{1}{2 \sqrt{\lambda_{1}}} e^{-\sqrt{\lambda_{1}}|x-y|}\right) w(t, y) d y \\
& \leq \frac{1}{\lambda_{1}}\left[\left(\chi_{2} \lambda_{2} \mu_{2}-\chi_{1} \lambda_{1} \mu_{1}\right)_{+}+\chi_{2} \mu_{2}\left(\lambda_{1}-\lambda_{2}\right)_{+}\right]\left(\bar{u}_{1}+\delta\right) \\
&-\frac{1}{\lambda_{1}}\left[\left(\chi_{2} \lambda_{2} \mu_{2}-\chi_{1} \lambda_{1} \mu_{1}\right)_{-}+\chi_{2} \mu_{2}\left(\lambda_{1}-\lambda_{2}\right)_{-}\right]\left(\underline{u}_{1}-\delta\right)
\end{aligned}
$$

and

$$
\begin{aligned}
& \chi_{2} \lambda_{2} v_{2}-\chi_{1} \lambda_{1} v_{1} \\
& =\left(\chi_{2} \lambda_{2} \mu_{2}-\chi_{1} \lambda_{1} \mu_{1}\right) \int_{-h(t)}^{0} \frac{1}{2 \sqrt{\lambda_{1}}} e^{-\sqrt{\lambda_{1}}|x-y|} w(t,-y) d y \\
& \quad+\chi_{2} \lambda_{2} \mu_{2} \int_{-h(t)}^{0}\left(\frac{1}{2 \sqrt{\lambda_{2}}} e^{-\sqrt{\lambda_{2}}|x-y|}-\frac{1}{2 \sqrt{\lambda_{1}}} e^{-\sqrt{\lambda_{1}}|x-y|}\right) w(t,-y) d y \\
& \quad+\left(\chi_{2} \lambda_{2} \mu_{2}-\chi_{1} \lambda_{1} \mu_{1}\right) \int_{0}^{h(t)} \frac{1}{2 \sqrt{\lambda_{1}}} e^{-\sqrt{\lambda_{1}}|x-y|} w(t, y) d y \\
& \quad+\chi_{2} \lambda_{2} \mu_{2} \int_{0}^{h(t)}\left(\frac{1}{2 \sqrt{\lambda_{2}}} e^{-\sqrt{\lambda_{2}}|x-y|}-\frac{1}{2 \sqrt{\lambda_{1}}} e^{-\sqrt{\lambda_{1}}|x-y|}\right) w(t, y) d y \\
& \geq \\
& \frac{1}{\lambda_{1}}\left[\left(\chi_{2} \lambda_{2} \mu_{2}-\chi_{1} \lambda_{1} \mu_{1}\right)_{+}+\chi_{2} \mu_{2}\left(\lambda_{1}-\lambda_{2}\right)_{+}\right]\left(\underline{u}_{1}-\delta\right) \\
& \quad-\frac{1}{\lambda_{1}}\left[\left(\chi_{2} \lambda_{2} \mu_{2}-\chi_{1} \lambda_{1} \mu_{1}\right)_{-}+\chi_{2} \mu_{2}\left(\lambda_{1}-\lambda_{2}\right)_{-}\right]\left(\bar{u}_{1}+\delta\right) .
\end{aligned}
$$

It follows from similar arguments used in the proof of inequalities (3.19) and (3.21) that

$$
\begin{aligned}
\limsup _{t \rightarrow \infty} u(t, x) & \leq \frac{1}{b+\chi_{2} \mu_{2}-\chi_{1} \mu_{1}}\left\{a+\frac{1}{\lambda_{1}}\left[\left(\chi_{2} \lambda_{2} \mu_{2}-\chi_{1} \lambda_{1} \mu_{1}\right)_{+}+\chi_{2} \mu_{2}\left(\lambda_{1}-\lambda_{2}\right)_{+}\right] \bar{u}_{1}\right\} \\
& \triangleq \bar{u}_{2}
\end{aligned}
$$


uniformly on any bounded subset of $[0,+\infty)$ and

$$
\begin{aligned}
\liminf _{t \rightarrow \infty} u(t, x) & \geq \frac{1}{b+\chi_{2} \mu_{2}-\chi_{1} \mu_{1}}\left\{a-\frac{1}{\lambda_{1}}\left[\left(\chi_{2} \lambda_{2} \mu_{2}-\chi_{1} \lambda_{1} \mu_{1}\right)_{-}+\chi_{2} \mu_{2}\left(\lambda_{1}-\lambda_{2}\right)_{-}\right] \bar{u}_{1}\right\} \\
& \triangleq \underline{u}_{2}
\end{aligned}
$$

uniformly on any bounded subset of $[0,+\infty)$.

By (3.39) and (3.43), we find that

$$
\begin{aligned}
\bar{u}_{1}- & \bar{u}_{2} \\
= & \bar{u}_{1}-\frac{1}{b+\chi_{2} \mu_{2}-\chi_{1} \mu_{1}}\left\{a+\frac{1}{\lambda_{1}}\left[\left(\chi_{2} \lambda_{2} \mu_{2}-\chi_{1} \lambda_{1} \mu_{1}\right)_{+}+\chi_{1} \mu_{1}\left(\lambda_{1}-\lambda_{2}\right)_{+}\right] \bar{u}_{1}\right\} \\
= & \frac{1}{b+\chi_{2} \mu_{2}-\chi_{1} \mu_{1}}\left\{\overline { u } _ { 1 } \left(b+\chi_{2} \mu_{2}-\chi_{1} \mu_{1}-\frac{1}{\lambda_{1}}\left[\left(\chi_{2} \lambda_{2} \mu_{2}-\chi_{1} \lambda_{1} \mu_{1}\right)_{+}\right.\right.\right. \\
& \left.\left.\left.+\chi_{1} \mu_{1}\left(\lambda_{1}-\lambda_{2}\right)_{+}\right]\right)-a\right\} \\
= & \frac{\bar{u}_{1}}{b+\chi_{2} \mu_{2}-\chi_{1} \mu_{1}}\left\{H_{1}-\frac{1}{\lambda_{1}}\left[\left(\chi_{2} \lambda_{2} \mu_{2}-\chi_{1} \lambda_{1} \mu_{1}\right)_{+}+\chi_{1} \mu_{1}\left(\lambda_{1}-\lambda_{2}\right)_{+}\right]\right\} \\
\geq & 0 .
\end{aligned}
$$

Since $\bar{u}_{1} \geq \frac{a}{b}$,

$$
\begin{aligned}
\underline{u}_{2}= & \frac{1}{b+\chi_{2} \mu_{2}-\chi_{1} \mu_{1}}\left\{a-\frac{1}{\lambda_{1}}\left[\left(\chi_{2} \lambda_{2} \mu_{2}-\chi_{1} \lambda_{1} \mu_{1}\right)_{-}+\chi_{1} \mu_{1}\left(\lambda_{1}-\lambda_{2}\right)_{-}\right] \bar{u}_{1}\right\} \\
\leq & \frac{1}{b+\chi_{2} \mu_{2}-\chi_{1} \mu_{1}}\left\{a+\frac{1}{\lambda_{1}}\left[\left(\chi_{2} \lambda_{2} \mu_{2}-\chi_{1} \lambda_{1} \mu_{1}\right)_{+}+\chi_{1} \mu_{1}\left(\lambda_{1}-\lambda_{2}\right)_{+}\right] \frac{a}{b}\right. \\
& \left.-\frac{1}{\lambda_{1}}\left[\left(\chi_{2} \lambda_{2} \mu_{2}-\chi_{1} \lambda_{1} \mu_{1}\right)_{-}+\chi_{1} \mu_{1}\left(\lambda_{1}-\lambda_{2}\right)_{-}\right] \bar{u}_{1}\right\} \\
\leq & \frac{1}{b+\chi_{2} \mu_{2}-\chi_{1} \mu_{1}}\left\{a+\frac{1}{\lambda_{1}}\left[\left(\chi_{2} \lambda_{2} \mu_{2}-\chi_{1} \lambda_{1} \mu_{1}\right)_{+}+\chi_{1} \mu_{1}\left(\lambda_{1}-\lambda_{2}\right)_{+}\right] \frac{a}{b}\right. \\
& \left.-\frac{1}{\lambda_{1}}\left[\left(\chi_{2} \lambda_{2} \mu_{2}-\chi_{1} \lambda_{1} \mu_{1}\right)_{-}+\chi_{1} \mu_{1}\left(\lambda_{1}-\lambda_{2}\right)_{-}\right] \frac{a}{b}\right\} \\
= & \frac{a+\frac{a}{b}\left(\chi_{2} \mu_{2}-\chi_{1} \mu_{1}\right)}{b+\chi_{2} \mu_{2}-\chi_{1} \mu_{1}}=\frac{a}{b} .
\end{aligned}
$$

Using inequality (3.38), we have

$$
\begin{aligned}
\underline{u}_{2}= & \frac{1}{b+\chi_{2} \mu_{2}-\chi_{1} \mu_{1}}\left\{a-\frac{1}{\lambda_{1}}\left[\left(\chi_{2} \lambda_{2} \mu_{2}-\chi_{1} \lambda_{1} \mu_{1}\right)_{-}+\chi_{1} \mu_{1}\left(\lambda_{1}-\lambda_{2}\right)_{-}\right] \bar{u}_{1}\right\} \\
= & \frac{1}{\left(b+\chi_{2} \mu_{2}-\chi_{1} \mu_{1}\right)\left(b+\chi_{2} \mu_{2}-\chi_{1} \mu_{1}-H_{2}\right)} \\
& \times\left\{a\left(b+\chi_{2} \mu_{2}-\chi_{1} \mu_{1}-H_{2}\right)-a \frac{1}{\lambda_{1}}\left[\left(\chi_{2} \lambda_{2} \mu_{2}-\chi_{1} \lambda_{1} \mu_{1}\right)_{-}+\chi_{1} \mu_{1}\left(\lambda_{1}-\lambda_{2}\right)_{-}\right]\right\} \\
= & \frac{a}{\left(b+\chi_{2} \mu_{2}-\chi_{1} \mu_{1}\right)\left(b+\chi_{2} \mu_{2}-\chi_{1} \mu_{1}-H_{2}\right)}
\end{aligned}
$$




$$
\begin{aligned}
& \times\left\{b+\chi_{2} \mu_{2}-\chi_{1} \mu_{1}-H_{2}-\frac{1}{\lambda_{1}}\left[\left(\chi_{2} \lambda_{2} \mu_{2}-\chi_{1} \lambda_{1} \mu_{1}\right)_{-}+\chi_{1} \mu_{1}\left(\lambda_{1}-\lambda_{2}\right)_{-}\right]\right\} \\
= & \frac{a}{\left(b+\chi_{2} \mu_{2}-\chi_{1} \mu_{1}\right)\left(b+\chi_{2} \mu_{2}-\chi_{1} \mu_{1}-H_{2}\right)} \\
& \times\left\{b+\chi_{2} \mu_{2}-\chi_{1} \mu_{1}-\left(\frac { 1 } { \lambda _ { 1 } } \left[\left(\chi_{2} \lambda_{2} \mu_{2}-\chi_{1} \lambda_{1} \mu_{1}\right)_{+}+\chi_{1} \mu_{1}\left(\lambda_{1}-\lambda_{2}\right)_{+}\right.\right.\right. \\
& \left.\left.+\frac{1}{\lambda_{1}}\left[\left(\chi_{2} \lambda_{2} \mu_{2}-\chi_{1} \lambda_{1} \mu_{1}\right)_{-}+\chi_{1} \mu_{1}\left(\lambda_{1}-\lambda_{2}\right)_{-}\right]\right)\right\} \\
= & \frac{a}{\left(b+\chi_{2} \mu_{2}-\chi_{1} \mu_{1}\right)\left(b+\chi_{2} \mu_{2}-\chi_{1} \mu_{1}-H_{2}\right)} \\
& \times\left\{b+\chi_{2} \mu_{2}-\chi_{1} \mu_{1}-\frac{1}{\lambda_{1}}\left[\left|\chi_{2} \lambda_{2} \mu_{2}-\chi_{1} \lambda_{1} \mu_{1}\right|+\chi_{1} \mu_{1}\left|\lambda_{1}-\lambda_{2}\right|\right]\right\} \\
> & 0=\underline{u}_{1} .
\end{aligned}
$$

Repeating the procedure of Case I, we obtain

$$
\lim _{t \rightarrow \infty} u(t, x)=\frac{a}{b}, \quad \lim _{t \rightarrow \infty} v_{i}(t, x)=\frac{\mu_{i}}{\lambda_{i}} \frac{a}{b}, \quad i=1,2
$$

uniformly on any bounded subset of $[0,+\infty)$.

Therefore, it follows from the results of Cases I and II that if

$$
\begin{aligned}
b+\chi_{2} \mu_{2}-\chi_{1} \mu_{1}> & \min \left\{\frac{1}{\lambda_{2}}\left(\left|\chi_{1} \mu_{1} \lambda_{1}-\chi_{2} \mu_{2} \lambda_{2}\right|+\chi_{1} \mu_{1}\left|\lambda_{1}-\lambda_{2}\right|\right),\right. \\
& \left.\frac{1}{\lambda_{1}}\left(\left|\chi_{1} \mu_{1} \lambda_{1}-\chi_{2} \mu_{2} \lambda_{2}\right|+\chi_{2} \mu_{2}\left|\lambda_{1}-\lambda_{2}\right|\right)\right\},
\end{aligned}
$$

then

$$
\lim _{t \rightarrow \infty} u(t, x)=\frac{a}{b}, \quad \lim _{t \rightarrow \infty} v_{i}(t, x)=\frac{\mu_{i}}{\lambda_{i}} \frac{a}{b}, \quad i=1,2
$$

uniformly on any bounded subset of $[0,+\infty)$.

\section{Criteria governing spreading and vanishing}

To discuss the criteria for spreading and vanishing as well as later application, we present a comparison principle which is similar to Lemma 3.5 of [7]. We omit the details here.

Lemma 4.1 (Comparison principle) Let $(w(t, x), \xi(t))$ be a solution of the following problem:

$$
\begin{cases}w_{t}-w_{x x}+\chi_{1} v_{1, x} w_{x}-\chi_{2} v_{2, x} w_{x}=\omega(A-B w), & t>0,0<x<\xi(t), \\ w=0, \quad \xi^{\prime}(t)=-\mu w_{x}(t, \xi(t)), & t>0, x=\xi(t), \\ w_{x}(t, 0)=0, & t>0,\end{cases}
$$


where $A$ and $B$ are positive constants. Assume $\bar{\xi}(t) \in C^{1}([0, \infty]), \bar{w} \in C\left(\bar{D}_{1}\right) \cap C^{1,2}\left(D_{1}\right)$ with $D_{1}=\left\{(t, x) \in \mathbb{R}^{2}, t>0,0<x<\bar{\xi}(t)\right\}, \xi(0)=\xi_{0}$ and $w(0, x)=w_{0}(x)$. Moreover, let $(\bar{w}, \bar{\xi})$ satisfy

$$
\begin{cases}\bar{w}_{t}-\bar{w}_{x x}+\chi_{1} v_{1, x} \bar{w}_{x}-\chi_{2} v_{2, x} \bar{w}_{x} \geq \bar{w}(A-B \bar{w}), & t>0,0<x<\bar{\xi}(t), \\ \bar{w}=0, \quad \bar{\xi}^{\prime}(t) \geq-\mu \bar{w}_{x}(t, \bar{\xi}(t)), & t>0, x=\bar{\xi}(t), \\ \bar{w}_{x}(t, 0) \leq 0, & t>0 .\end{cases}
$$

If

$$
\xi_{0} \leq \bar{\xi}(0) \quad \text { and } \quad w_{0}(x) \leq \bar{w}(0, x) \quad \text { in }\left[0, \xi_{0}\right]
$$

then

$$
\xi(t) \leq \bar{\xi}(t) \quad \text { in }[0, \infty), \quad w(t, x) \leq \bar{w}(t, x) \quad \text { for } t \in[0, \infty) \text { and } x \in(0, \xi(t)) .
$$

Then, we first give a necessary condition for vanishing.

Theorem 4.2 Suppose that (2.4) holds. Let $\left(u, v_{1}, v_{2}, h\right)$ be any solution of (1.1). If $h_{\infty}<\infty$ then $h_{\infty} \leq \frac{\pi}{2} \sqrt{\frac{1}{a}}$. Hence, $h_{0} \geq \frac{\pi}{2} \sqrt{\frac{1}{a}}$ implies $h_{\infty}=\infty$ due to $h^{\prime}(t)>0$ for $t>0$.

Proof By Theorem 3.3, if $h_{\infty}<\infty$ then

$$
\lim _{t \rightarrow \infty}\|u(t, \cdot)\|_{C([0, h(t)])}=0, \quad \lim _{t \rightarrow \infty} v_{i}(t, x)=0, \quad i=1,2 \text {, uniformly on }[0,+\infty) .
$$

We assume $h_{\infty}>\frac{\pi}{2} \sqrt{\frac{1}{a}}$ to get a contradiction. Let

$$
a^{\varepsilon}:=a-\chi_{1} \lambda_{1} \varepsilon<a .
$$

It is clear that $a^{\varepsilon} \rightarrow a$ as $\varepsilon \rightarrow 0$. Thus there exists a positive constant $\varepsilon_{0}$ such that

$$
h_{\infty}>\frac{\pi}{2} \sqrt{\frac{1}{a^{\varepsilon}}}, \quad \forall \varepsilon<\varepsilon_{0} .
$$

For any $\varepsilon<\varepsilon_{0}$, due to (4.2), there exists a $\tau \gg 1$ such that

$$
\begin{aligned}
& v_{i}(t, x) \leq \varepsilon, \quad u(t, x) \leq \varepsilon, \quad \forall t \geq \tau, x \in\left[0, h_{\infty}\right], \\
& h(\tau)>\max \left\{h_{0}, \frac{\pi}{2} \sqrt{\frac{1}{a^{\varepsilon}}}\right\} .
\end{aligned}
$$

Set $l=h(\tau)$. Then $l>\frac{\pi}{2} \sqrt{\frac{1}{a^{\varepsilon}}}$. Therefore, we have

$$
u_{t}-u_{x x}+\chi_{1} v_{1, x} u_{x}-\chi_{2} v_{2, x} u_{x} \geq u\left[a-\chi_{1} \lambda_{1} \varepsilon-\left(b+\chi_{2} \mu_{2}-\chi_{1} \mu_{1}\right) u\right] .
$$


Let $\omega$ be the positive solution of the following initial boundary value problem with a fixed boundary:

$$
\begin{cases}\omega_{t}-\omega_{x x}+\chi_{1} v_{1, x} \omega_{x}-\chi_{2} v_{2, x} \omega_{x} & \\ \quad=\omega\left[a-\chi_{1} \lambda_{1} \varepsilon-\left(b+\chi_{2} \mu_{2}-\chi_{1} \mu_{1}\right) \omega\right], & t>\tau, 0<x<l, \\ \omega_{x}(t, 0)=0, \quad \omega(t, l)=0, & t>\tau, \\ \omega(\tau, x)=u(\tau, x), & 0<x<l .\end{cases}
$$

By the comparison principle,

$$
\omega(t, x) \leq u(t, x) \quad \text { for } t \geq \tau, 0 \leq x \leq l
$$

Since $a^{\varepsilon}>\left(\frac{\pi}{2 l}\right)^{2}$, it is well known that $\omega(t, x) \rightarrow \theta(x)$ as $t \rightarrow \infty$ uniformly on any compact subset of $(0, l)$, where $\theta$ is the unique positive solution of

$$
\left\{\begin{array}{l}
-\theta^{\prime \prime}=\theta\left[a^{\varepsilon}-\left(b+\chi_{2} \mu_{2}-\chi_{1} \mu_{1}\right) \theta\right], \quad 0<x<l, \\
\theta^{\prime}(0)=0, \quad \theta(l)=0 .
\end{array}\right.
$$

Hence, $\liminf _{t \rightarrow \infty} u(t, x) \geq \lim _{t \rightarrow \infty} \omega(t, x)=\theta(x)>0$ in $(0, l)$. This is a contradiction to (4.2). Thus, $h_{\infty} \leq \frac{\pi}{2} \sqrt{\frac{1}{a}}$. Due to $h^{\prime}(t)>0$ for $t>0, h_{0} \geq \frac{\pi}{2} \sqrt{\frac{1}{a}}$ implies $h_{\infty}=\infty$.

Now we discuss the case $h_{0}<\frac{\pi}{2} \sqrt{\frac{1}{a}}$.

Theorem 4.3 Suppose $b>\chi_{1} \mu_{1}-\chi_{2} \mu_{2}+H$ and $h_{0}<\frac{\pi}{2} \sqrt{\frac{1}{a}}$. Then there exists $a \bar{\mu}>0$ depending only on $u_{0}(x)$ such that $h_{\infty}=+\infty$ when $\mu \geq \bar{\mu}$.

Proof The proof here is inspired by Lemma 3.2 in [40]. By Lemma 2.1, we have $0<u<R_{1}$ and so there exists a constant $\delta^{*}>0$ satisfying

$$
u\left[a-\left(\chi_{2} \mu_{2}+b-\chi_{1} \mu_{1}\right) u-\chi_{1} \lambda_{1} v_{1}+\chi_{2} \lambda_{2} v_{2}\right] \geq-\delta^{*} u
$$

Now, we consider the auxiliary free boundary problem

$$
\begin{cases}w_{t}-w_{x x}+\chi_{1} v_{1, x} w_{x}-\chi_{2} v_{2, x} w_{x}=-\delta^{*} w, & t \geq 0,0<x<r(t), \\ w_{x}(t, 0)=0, \quad w(t, r(t))=0, & t \geq 0, \\ w(0, x)=u_{0}(x), & 0 \leq x \leq r(0)=h_{0} .\end{cases}
$$

It is easy to see that (4.3) admits a unique solution $(w(t, x), r(t))$ which is well defined for all $t>0$. Moreover, $r^{\prime}(t)>0$ for $t>0$. Similar to the proof of Lemma 4.1 in [32], we have

$$
u(t, x) \geq w(t, x), \quad h(t) \geq r(t), \quad \forall t \geq 0, x \in[0, r(t)]
$$

In what follows, we are going to prove that for all large $\mu$,

$$
r(2) \geq \pi \sqrt{\frac{1}{a}}
$$


To this end, we first choose a smooth function $\underline{r}(t)$ with $\underline{r}(0)=\frac{h_{0}}{2}, \underline{r}^{\prime}(t)>0$ and $\underline{r}(2)=\pi \sqrt{\frac{1}{a}}$. We then consider the following initial-boundary value problem:

$$
\begin{cases}\underline{w}_{t}-\underline{w}_{x x}+\chi_{1} v_{1, x} \underline{w}_{x}-\chi_{2} v_{2, x} \underline{w}_{x}=-\delta^{*} \underline{w}, & t \geq 0,0<x<\underline{r}(t), \\ \underline{w}_{x}(t, 0)=0, \quad \underline{w}(t, \underline{r}(t))=0, & t \geq 0, \\ \underline{w}(0, x)=\underline{u}_{0}(x), & 0 \leq x \leq \frac{h_{0}}{2} .\end{cases}
$$

Here, for a smooth initial value $\underline{u}_{0}$, we require

$$
0<\underline{u}_{0}(x) \leq u_{0}(x) \quad \text { for } x \in\left[0, \frac{h_{0}}{2}\right], \quad \underline{w}_{0}^{\prime}(0)=\underline{w}_{0}\left(\frac{h_{0}}{2}\right)=0, \quad \underline{w}_{0}^{\prime}\left(\frac{h_{0}}{2}\right)<0 .
$$

The standard theory for parabolic equations ensures that (4.6) has a unique positive solution $\underline{w}$ and $\underline{w}_{x}(t, \underline{r}(t))<0$ for all $t \in[0,2]$ due to Hopf's Lemma. According to our choice of $\underline{r}(t)$ and $\underline{w}_{0}(x)$, there is a constant $\bar{\mu}>0$ such that for all $\mu \geq \bar{\mu}$,

$$
\underline{r}^{\prime}(t) \leq-\mu \underline{w}_{x}(t, \underline{r}(t)), \quad \forall 0 \leq t \leq 2
$$

Note that $\underline{r}(0)=\frac{h_{0}}{2}<r(0)$, so it follows from (4.3), (4.6), (4.7) and (4.8) that

$$
w(t, x) \geq \underline{w}(t, x), \quad r(t) \geq \underline{r}(t), \quad \forall t \in[0,2], x \in[0, \underline{r}(t)],
$$

which particularly implies $r(2) \geq \underline{r}(2)=\pi \sqrt{\frac{1}{a}}$, and so (4.5) holds. Hence, in view of (4.4) and (4.5), we have

$$
h_{\infty}=\lim _{t \rightarrow \infty} h(t)>h(2) \geq \pi \sqrt{\frac{1}{a}} .
$$

Theorem 4.2 now yields the desired result.

Lemma 4.4 For every $l>0$, let $\lambda^{1}(l)$ and $\Psi(y)$ be the first eigenvalue and corresponding eigenfunction of the problem

$$
\left\{\begin{array}{l}
-\psi_{y y}+Q \psi_{y}=\lambda \psi, \quad 0<y<l \\
\psi_{y}(0)=0, \quad \psi(l)=0
\end{array}\right.
$$

with $Q$ a positive constant and $\|\Psi\|_{L^{2}((0, l))}=1$. Then we have the following conclusions:

(i) $\Psi(y)>0$ and $\Psi_{y}(y)<0$ for $0<y<l$.

(ii) $\lambda^{1}$ is a strictly decreasing and continuous function in $l$, and

$$
\lim _{l \rightarrow 0^{+}} \lambda^{1}(l)=+\infty, \quad \lim _{l \rightarrow+\infty} \lambda^{1}(l)=\frac{Q^{2}}{4} .
$$

Thus, there exists an unique constant $L>0$ such that

$$
\lambda^{1}(L)=a+\chi_{2} \mu_{2} R_{1}+\frac{Q^{2}}{4}
$$


and

$$
\lambda^{1}(l)<a+\chi_{2} \mu_{2} R_{1}+\frac{Q^{2}}{4} \quad \text { for } l>L \quad \text { and } \quad \lambda^{1}(l)>a+\chi_{2} \mu_{2} R_{1}+\frac{Q^{2}}{4} \quad \text { for } l<L,
$$

where $R_{1}$ is defined in Lemma 2.1.

(iii) The constant $L$ satisfies $L<\frac{\pi}{2} \sqrt{\frac{1}{a}}$.

Proof First, a general solution of the ODE $-\psi_{y y}+Q \psi_{y}=\lambda \psi$ has the form

$$
\psi(y)=\exp \left(\frac{Q}{2} y\right)\left[C_{1} \cos \left(\frac{\sqrt{4 \lambda-Q^{2}}}{2} y\right)+C_{2} \sin \left(\frac{\sqrt{4 \lambda-Q^{2}}}{2} y\right)\right]
$$

with $4 \lambda-Q^{2}>0$. Note that $\psi_{y}(0)=0$ and $\psi(l)=0$, direct calculations yield

$$
\left\{\begin{array}{l}
C_{2} \frac{Q}{2}+C_{2} \frac{\sqrt{4 \lambda-Q^{2}}}{2}=0, \\
C_{1} \cos \left(\frac{\sqrt{4 \lambda-Q^{2}}}{2} l\right)+C_{2} \sin \left(\frac{\sqrt{4 \lambda-Q^{2}}}{2} l\right)=0 .
\end{array}\right.
$$

From (4.9), it is easy to see that, either $C_{1}=C_{2}=0$ or $C_{1} \neq 0, C_{2} \neq 0$. If $C_{1}=C_{2}=0$, we have $\psi(y) \equiv 0$, which is impossible for the eigenfunction. From (4.9), $C_{1} \neq 0$ and $C_{2} \neq 0$ imply that

$$
\frac{\sqrt{4 \lambda-Q^{2}}}{2} l=\arctan \left(\frac{\sqrt{4 \lambda-Q^{2}}}{Q}\right)+n \pi \quad \text { for } n=0,1,2, \ldots
$$

Thus the first eigenvalue $\lambda^{1}(l)$ satisfies

$$
\begin{aligned}
\lambda^{1}(l) & =\frac{1}{4}\left[Q^{2}+\frac{4}{l^{2}}\left(\arctan \left(\frac{\sqrt{4 \lambda-Q^{2}}}{Q}\right)\right)^{2}\right] \\
& <\frac{1}{4}\left[Q^{2}+\frac{4}{l^{2}}\left(\frac{\pi}{2}\right)^{2}\right]=\frac{Q^{2}}{4}+\frac{\pi^{2}}{4 l^{2}} .
\end{aligned}
$$

Hence the corresponding eigenfunction satisfies

$$
\Psi(y)=\mathcal{C} \exp \left(\frac{Q}{2} y\right)\left[\frac{\sqrt{4 \lambda^{1}-Q^{2}}}{Q} \cos \left(\frac{\sqrt{4 \lambda^{1}-Q^{2}}}{2} y\right)-\sin \left(\frac{\sqrt{4 \lambda^{1}-Q^{2}}}{2} y\right)\right]
$$

where $\mathcal{C}$ is a positive constant to guarantee $\|\Psi(y)\|_{L^{2}((0 . l))}=1$. Form (4.10), it is easy to deduce that

$$
0<\frac{\sqrt{4 \lambda^{1}-Q^{2}}}{2} y<\arctan \left(\frac{\sqrt{4 \lambda^{1}-Q^{2}}}{Q}\right) \quad \text { for } 0<y<l,
$$

which implies $\Psi(y)>0$ for $0<y<l$. Direct calculation gives

$$
\begin{aligned}
\Psi_{y}(y)= & \mathcal{C} \exp \left(\frac{Q}{2} y\right)\left[\frac{Q}{2} \frac{\sqrt{4 \lambda^{1}-Q^{2}}}{Q} \cos \left(\frac{\sqrt{4 \lambda^{1}-Q^{2}}}{2} y\right)-\frac{Q}{2} \sin \left(\frac{\sqrt{4 \lambda^{1}-Q^{2}}}{2} y\right)\right. \\
& -\frac{\sqrt{4 \lambda^{1}-Q^{2}}}{Q} \frac{\sqrt{4 \lambda^{1}-Q^{2}}}{2} \sin \left(\frac{\sqrt{4 \lambda^{1}-Q^{2}}}{2} y\right)
\end{aligned}
$$




$$
\begin{aligned}
& \left.-\frac{\sqrt{4 \lambda^{1}-Q^{2}}}{2} \cos \left(\frac{\sqrt{4 \lambda^{1}-Q^{2}}}{2} y\right)\right] \\
= & -\mathcal{C} \exp \left(\frac{Q}{2} y\right)\left[\frac{Q}{2} \sin \left(\frac{\sqrt{4 \lambda^{1}-Q^{2}}}{2} y\right)+\frac{4 \lambda^{1}-Q^{2}}{2 Q} \sin \left(\frac{\sqrt{4 \lambda^{1}-Q^{2}}}{2} y\right)\right] \\
< & 0 \quad \text { for } 0<y<l .
\end{aligned}
$$

Thus (i) is satisfied. With the help of (4.10), the proof of (ii) is similar to that of Theorem 3.1 in [52], and we omit it here. Using (ii) and (4.10) again, we have

$$
\lambda^{1}(L)=a+\chi_{2} \mu_{2} R_{1}+\frac{Q^{2}}{4}<\frac{\pi^{2}}{4 L^{2}}+\frac{Q^{2}}{4}
$$

which implies $L<\frac{\pi}{2} \sqrt{\frac{1}{a}}$ immediately. Thus (iii) is satisfied.

Theorem 4.5 Suppose $b>\chi_{1} \mu_{1}-\chi_{2} \mu_{2}+H$ and $h_{0}<L$, where $L<\frac{\pi}{2} \sqrt{\frac{1}{a}}$ is a constant defined in Lemma 4.4. Then there exists a $\underline{\mu}>0$, depending on $u_{0}(x)$, such that $h_{\infty}<\infty$ when $\mu \leq \underline{\mu}$.

Proof Inspired by [7], we are going to construct a suitable upper solution to (1.1) and then apply the comparison principe.

For any given $T>0$, in view of (2.29), there exists a positive constant $Q$ such that

$$
\left|\chi_{1} v_{1, x}-\chi_{2} v_{2, x}\right| \leq Q \quad \text { for } 0<t<T, x \in \mathbb{R}^{+}
$$

Let $\lambda^{1}\left(h_{0}\right)$ and $\Psi(y)$ be the eigenvalue and corresponding eigenfunction of the problem

$$
\left\{\begin{array}{l}
-\Psi_{y y}+Q \Psi_{y}=\lambda^{1}\left(h_{0}\right) \Psi, \quad 0<y<h_{0} \\
\Psi_{y}(0)=0, \quad \Psi\left(h_{0}\right)=0
\end{array}\right.
$$

with $\|\Psi\|_{L^{2}((0, l))}=1$. Due to Lemma 4.4(i), we also obtain

$$
\Psi(y)>0 \quad \text { and } \quad \Psi_{y}(y)<0 \quad \text { for } 0<y<h_{0} .
$$

Using $h_{0}<L$ and Lemma 4.4(ii), we obtain

$$
\lambda^{1}\left(h_{0}\right)>a+\chi_{2} \mu_{2} R_{1}+\frac{Q^{2}}{4}
$$

for $t \geq 0$, and define

$$
\begin{aligned}
& \sigma(t)=h_{0}\left(1+\delta-\frac{\delta}{2} e^{-\gamma t}\right), \quad t \geq 0, \\
& \bar{u}(t, x)=P e^{-\gamma t} \Psi\left(\frac{x h_{0}}{\sigma(t)}\right) \quad \text { for } t \geq 0 \text { and } 0 \leq x \leq \sigma(t),
\end{aligned}
$$


in which $\delta, \gamma, P$ are positive constants that will be determined later. It is easy to see that $\bar{u}(t, \sigma(t))=0$ and

$$
\bar{u}(0, x)=P \Psi\left(\frac{x}{1+\frac{\delta}{2}}\right) \geq\left\|u_{0}\right\|_{\infty} \geq u_{0}(x)
$$

provided $P>0$ is sufficiently large. Note that

$$
h_{0}\left(1+\frac{\delta}{2}\right) \leq \sigma(t) \leq h_{0}(1+\delta) \quad \text { and } \quad \sigma^{\prime}(t)=\frac{h_{0} \delta}{2} \gamma e^{-\gamma t}
$$

Then, direct calculations yield

$$
\begin{aligned}
\bar{u}_{t}- & \bar{u}_{x x}+\chi_{1} v_{1, x} \bar{u}_{x}-\chi_{2} v_{2, x} \bar{u}_{x}-\bar{u}\left[a+\chi_{2} \mu_{2} R_{1}-\left(b+\chi_{2} \mu_{2}-\chi_{1} \mu_{1}\right) \bar{u}\right] \\
= & P e^{-\gamma t}\left[-\gamma \Psi-\frac{y h_{0} \sigma^{\prime}(t)}{\sigma^{2}(t)} \Psi_{y}-\frac{h_{0}^{2}}{\sigma^{2}(t)} \Psi_{y y}+\frac{\chi_{1} v_{1, x} h_{0}}{\sigma(t)} \Psi_{y}\right. \\
& \left.-\frac{\chi_{2} v_{2, x} h_{0}}{\sigma(t)} \Psi_{y}-\left(a+\chi_{2} \mu_{2} R_{1}\right) \Psi\right]+\left(b+\chi_{2} \mu_{2}-\chi_{1} \mu_{1}\right) \bar{u}^{2} \\
\geq & P e^{-\gamma t}\left[-\gamma \Psi+\frac{h_{0}^{2}\left(\lambda_{0}\left(h_{0}\right) \Psi-Q \Psi_{y}\right)}{\sigma^{2}(t)}+\frac{\chi_{1} v_{1, x} h_{0}}{\sigma(t)} \Psi_{y}-\frac{\chi_{2} v_{2, x} h_{0}}{\sigma(t)} \Psi_{y}-\left(a+\chi_{2} \mu_{2} R_{1}\right) \Psi\right] \\
\geq & P e^{-\gamma t}\left[-\gamma \Psi+\left(\frac{\lambda_{0}\left(h_{0}\right)}{(1+\delta)^{2}}-a-\chi_{2} \mu_{2} R_{1}\right) \Psi+\left(\chi_{1} v_{1, x}-\chi_{2} v_{2, x}-Q \frac{1}{1+\delta}\right) \frac{\Psi_{y} h_{0}}{\sigma(t)}\right]
\end{aligned}
$$

for all $0<t<T$ and $0<x<\sigma(t)$. By (4.11) and (4.13), we choose $\delta>0$ small such that

$$
\begin{aligned}
& \chi_{1} v_{1, x}-\chi_{2} v_{2, x}-Q \frac{1}{1+\delta}<0 \quad \text { and } \\
& \frac{\lambda_{0}\left(h_{0}\right)}{(1+\delta)^{2}}-a-\chi_{2} \mu_{2} R_{1} \geq \frac{1}{2}\left[\lambda_{0}\left(h_{0}\right)-a-\chi_{2} \mu_{2} R_{1}\right] .
\end{aligned}
$$

Choosing $\gamma=\frac{1}{4}\left[\lambda_{0}\left(h_{0}\right)-a-\chi_{2} \mu_{2} R_{1}\right]$, in view of (4.14), we have

$$
\bar{u}_{t}-\bar{u}_{x x}+\chi_{1} v_{1, x} \bar{u}_{x}-\chi_{2} v_{2, x} \bar{u}_{x} \geq \bar{u}\left[a+\chi_{2} \mu_{2} R_{1}-\left(b+\chi_{2} \mu_{2}-\chi_{1} \mu_{1}\right) \bar{u}\right] .
$$

We calculate

$$
\begin{aligned}
& \sigma^{\prime}(t)=\frac{h_{0} \delta}{2} \gamma e^{-\gamma t} \\
& -\mu \bar{u}_{x}(t, \sigma(t))=-\mu P e^{-\gamma t}\left(\frac{h_{0}}{\sigma(t)} \Psi_{y}\left(h_{0}\right)\right) \leq \mu P e^{-\gamma t} \frac{\left|\Psi_{y}\left(h_{0}\right)\right|}{1+\frac{\delta}{2}} .
\end{aligned}
$$

Hence, if we take

$$
\underline{\mu} \triangleq \frac{h_{0} \delta \gamma(2+\delta)}{4 P\left|\Psi_{y}\left(h_{0}\right)\right|}
$$

then for any $0<\mu \leq \underline{\mu}$,

$$
\sigma^{\prime}(t) \geq-\mu \bar{u}_{x}(t, \sigma(t))
$$


Therefore, for our choice of $\delta, \gamma, P$ and $\underline{\mu},(\bar{u}(t, x), \sigma(t))$ satisfies

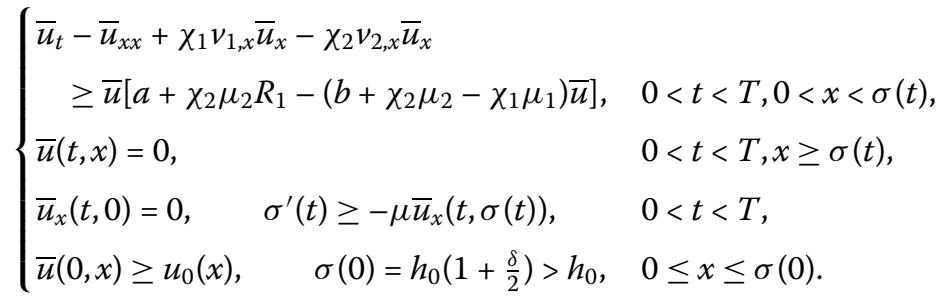

Thus, we can apply Lemma 4.1 to conclude that $h(t) \leq \sigma(t)$ and $u(t, x) \leq \bar{u}(t, x)$ for $0<t<T$ and $0 \leq x \leq h(t)$. Since $T$ can be arbitrarily large, we deduce that $h_{\infty} \leq \lim _{t \rightarrow \infty} \sigma(t)=$ $h_{0}(1+\delta)<\infty$ for all $\mu \leq \mu$.

\section{Conclusion}

In summary, we considered an attraction-repulsion chemotaxis system with a free boundary in one space dimension. In model (1.1), the so-called free boundary $x=h(t)$ characterizes the change of the expanding front for the mobile species. Our conclusions not only provide sufficient conditions for species spreading success and spreading failure, but also the long time behavior of the mobile species, chemo-attraction and chemo-repulsion. Precisely, we prove a spreading-vanishing dichotomy for this model, that is, either the species fails to establish and vanishes eventually, or the species successfully spreads to infinity as $t \rightarrow \infty$ and stabilizes at a constant equilibrium state under some sufficient conditions. Not only that, we also discuss the criteria for spreading and vanishing. These analytical findings disclose that the change of invasion region to species can determine whether the invasion is successful or not.

The free boundary in model (1.1) describes a one-dimensional environment. We realize that the two- or three-dimensional case better matches reality. However, there will be more challenges in both mathematical analysis and numerical simulation for models with multidimensional free boundary. A promising extension is to explicitly consider the asymptotic spreading speed of the invasion species.

Acknowledgements

Not applicable.

Funding

The work is partially supported by National Natural Science Foundation of China (11771380 and 11401515).

Availability of data and materials

Data sharing not applicable to this article as no data sets were generated or analyzed during the current study.

Competing interests

The authors declare that they have no competing interests.

Authors' contributions

All authors contributed equally to the writing of this paper. All authors read and approved the final manuscript.

\section{Publisher's Note}

Springer Nature remains neutral with regard to jurisdictional claims in published maps and institutional affiliations. 


\section{References}

1. Bellomo, N., Bellouquid, A., Tao, T., Winkler, M.: Toward a mathematical theory of Keller-Segel models of pattern formation in biological tissues. Math. Models Methods Appl. Sci. 25(9), 1663-1763 (2015)

2. Bunting, G., Du, Y.H., Krakowski, K.: Spreading speed revisited: analysis of a free boundary model (special issue dedicated to H. Matano). Netw. Heterog. Media 7(4), 583-603 (2012)

3. Chen, X.F., Friedman, A.: A free boundary problem arising in a model of wound healing. SIAM J. Math. Anal. 32(4), 778-800 (2000)

4. Du, Y.H., Guo, Z.M.: Spreading-vanishing dichotomy in the diffusive logistic model with a free boundary II. J. Differ. Equ. 250(12), 4336-4366 (2011)

5. Du, Y.H., Guo, Z.M., Peng, R.: A diffusive logistic model with a free boundary in time-periodic environment. J. Funct. Anal. 265(9), 2089-2142 (2013)

6. Du, Y.H., Liang, X.: Pulsating semi-waves in periodic media and spreading speed determined by a free boundary model. Ann. Inst. Henri Poincaré, Anal. Non Linéaire 32(2), 279-305 (2015)

7. Du, Y.H., Lin, Z.G.: Spreading-vanishing dichotomy in the diffusive logistic model with a free boundary. SIAM J. Math. Anal. 42(1), 337-405 (2010)

8. Du, Y.H., Lin, Z.G.: The diffusive competition model with a free boundary: invasion of a superior of inferior competitor. Discrete Contin. Dyn. Syst., Ser. B 19(10), 3105-3132 (2014)

9. Du, Y.H., Lou, B.D.: Spreading and vanishing in nonlinear diffusion problems with free boundaries. J. Eur. Math. Soc 17(10), 2673-2724 (2015)

10. Du, Y.H., Wang, M.X., Zhou, M.L.: Semi-wave and spreading speed for the diffusive competition model with a free boundary. J. Math. Pures Appl. 107(3), 253-287 (2017)

11. Ge, J., Kim, K.I., Lin, Z.G., Zhu, H.P.: An SIS reaction-diffusion-advection model in a low-risk and high-risk domain J. Differ. Equ. 259(10), 5486-5509 (2015)

12. Guo, J.S., Wu, C.H.: On a free boundary problem for a two-species weak competition system. J. Dyn. Differ. Equ. 24(4), 873-895 (2012)

13. Guo, J.S., Wu, C.H.: Dynamics for a two-species competition-diffusion model with two free boundaries. Nonlinearity 28(1), 1-27 (2015)

14. Jin, H.Y.: Boundedness of the attraction-repulsion Keller-Segel system. J. Math. Anal. Appl. 422(2), 1463-1478 (2015)

15. Kaneko, Y., Yanmada, Y.: A free boundary problem for a reaction-diffusion equation appearing in ecology. Adv. Math. Sci. Appl. 21(2), 467-492 (2011)

16. Keller, E.F., Segel, L.A.: Initiation of slime mold aggregation viewed as an instability. J. Theor. Biol. 26(3), 399-415 (1970)

17. Keller, E.F., Segel, L.A.: A model for chemotaxis. J. Theor. Biol. 30, 225-234 (1971)

18. Kim, K.I., Lin, Z.G., Zhang, Q.Y.: An SIR epidemic model with free boundary. Nonlinear Anal., Real World Appl. 14(5), 1992-2001 (2013)

19. Lin, Z.G., Zhu, H.P.: Spatial spreading model and dynamics of West Nile virus in birds and mosquitoes with free boundary. J. Math. Biol. 75, 1381-1409 (2017)

20. Liu, J., Shi, J., Wang, Z.A.: Classical solutions and steady states of an attraction-repulsion chemotaxis in one dimension. J. Biol. Dyn. 6(1), 31-41 (2012)

21. Liu, K., Mu, C., Gao, Y.: Boundedness and blow up in the higher-dimensional attraction-repulsion chemotaxis with non-linear diffusion. J. Differ. Equ. 261(8), 4524-4572 (2016)

22. Mimura, M., Yamada, Y., Yotsutani, S.: A free boundary problem in ecology. Jpn. J. Appl. Math. 2(1), 151-186 (1985)

23. Mimura, M., Yamada, Y., Yotsutani, S.: Stability analysis for free boundary problems in ecology. Hiroshima Math. J. 16(3), 477-498 (1986)

24. Nagai, T:: Global existence and blow up of solution to a chemotaxis system. Nonlinear Anal. 47(2), 777-787 (2001)

25. Nagai, T., Senba, T., Yoshida, S.: Application of the Trudinger-Moser inequality to a parabolic system of chemotaxis. Funkc. Ekvacioj 40(3), 411-433 (1997)

26. Rubinstein, LI: The Stefan Problem. Am. Math. Soc, Providence (1971)

27. Salako, R.B., Shen, W.: Global existence and asymptotic behavior of classical solutions to a parabolic-elliptic chemotaxis system with logistic source on $\mathbb{R}^{N}$. J. Dyn. Differ. Equ. 262(11), 5635-5690 (2017)

28. Salako, R.B., Shen, W.: Global classical solutions, stability equilibria, and spreading speeds in attraction-repulsion chemotaxis systems with logistic source on $\mathbb{R}^{N}$. arXiv:1612.00924v3

29. Salako, R.B., Shen, W.: Existence of traveling wave solutions to parabolic-elliptic-elliptic chemotaxis systems with logistic source. arXiv:1701.02633v2

30. Tarboush Abdelrazig, K., Lin, Z.G., Zhang, M.Y.: Spreading and vanishing in a West Nile virus model with expanding fronts. Sci. China Math. 60(5), 841-860 (2017)

31. Wang, J.P., Wang, M.X.: The diffusive Beddington-DeAngelis predator-prey model with nonlinear prey-taxis and free boundary. Math. Methods Appl. Sci. 41(16), 1-22 (2018)

32. Wang, M.X.: On some free boundary problems of the prey-predator model. J. Differ. Equ. 256(10), 3365-3394 (2014)

33. Wang, M.X.: The diffusive logistic equation with a free boundary and sign-changing coefficient. J. Differ. Equ. 258(4), $1252-1266(2015)$

34. Wang, M.X.: Spreading and vanishing in the diffusive prey-predator model with a free boundary. Commun. Nonlinear Sci. Numer. Simul. 23(1-3), 311-327 (2015)

35. Wang, M.X.: A diffusive logistic equation with a free boundary and sign-changing coefficient in time-periodic environment. J. Funct. Anal. 270(2), 483-508 (2016)

36. Wang, M.X.: Existence and uniqueness of solutions of free boundary problems in heterogeneous environments. Discrete Contin. Dyn. Syst., Ser. B (2018). https://doi.org/10.3924/dcdsb.2018179

37. Wang, M.X., Zhang, Q.Y.: Dynamics for the diffusive Leslie-Gower model with double free boundaries. Discrete Contin. Dyn. Syst. 38(5), 2591-2607 (2018)

38. Wang, M.X., Zhang, Y.: Note on a two-species competition-diffusion model with two free boundaries. Nonlinear Anal. TMA 159, 458-467 (2017)

39. Wang, M.X., Zhang, Y.: Dynamic for a diffusive prey-predator model with different free boundaries. J. Differ. Equ. 264, $3527-3558(2018)$ 
40. Wang, M.X., Zhao, J.F.: Free boundary problems for a Lotka-Volterra competition model. J. Dyn. Differ. Equ. 26(3), 655-672 (2014)

41. Wang, M.X., Zhao, J.F.: A free boundary problem for a predator-prey model with double free boundaries. J. Dyn. Differ Equ. 29(3), 957-979 (2017)

42. Wang, M.X., Zhao, Y.G.: A semilinear parabolic system with a free boundary. Z. Angew. Math. Phys. 66, 3309-3332 (2015)

43. Wang, Y., Xiang, Z.Y.: Boundedness in a quasilinear $2 D$ parabolic-parabolic attraction-repulsion chemotaxis system. Discrete Contin. Dyn. Syst., Ser. B 21(6), 1953-1973 (2016)

44. Wang, Y.L.: Global bounded weak solutions to a degenerate quasilinear attraction repulsion chemotaxis system with rotation. Comput. Math. Appl. 72(9), 2226-2240 (2016)

45. Zhang, Q.S., Li, Y.X.: An attraction-repulsion chemotaxis system with logistic source. Z. Angew. Math. Mech. 96(5), 570-584 (2016)

46. Zhao, J.F., Wang, M.X.: A free boundary problem of a predator-prey model with higher dimension and heterogeneous environment. Nonlinear Anal., Real World Appl. 16(7), 250-263 (2014)

47. Zhao, Y.G., Wang, M.X.: Free boundary problems for the diffusive competition system in higher dimension with sign-changing coefficients. IMA J. Appl. Math. 81(2), 255-280 (2016)

48. Zhao, Y.L., Liu, Z.H., Zhou, L.: Dynamic for a nonlocal reaction-diffusion population model with a free boundary. Acta Appl. Math. (2018). https://doi.org/10.1007/s10440-018-0188-8

49. Zhao, Y.L., Liu, Z.H., Zhou, L.: Dynamic for a nonlocal competition system with a free boundary. Appl. Anal. (2018). https://doi.org/10.1080/00036811.2018.1466282

50. Zheng, P., Mu, C., Hu, X.: Boundedness in the higher dimensional attraction-repulsion chemotaxis-growth system. Comput. Math. Appl. 72(9), 2194-2202 (2016)

51. Zhou, L., Zhang, S., Liu, Z.H.: A free boundary problem of a predator-prey model with advection in heterogeneous environment. Appl. Math. Comput. 289, 22-36 (2016)

52. Zhou, L., Zhang, S., Liu, Z.H.: A reaction-diffusion-advection equation with a free boundary and sign-changing coefficient. Acta Appl. Math. 143, 189-216 (2016)

\section{Submit your manuscript to a SpringerOpen ${ }^{\circ}$ journal and benefit from:}

- Convenient online submission

- Rigorous peer review

- Open access: articles freely available online

- High visibility within the field

- Retaining the copyright to your article

Submit your next manuscript at $\gg$ springeropen.com 